\title{
Anionic, in situ Generation of Formaldehyde. A Very Useful and Versatile Tool in Synthesis.
}

\section{Geoffrey Deguest, Laurent Bischoff*, Corinne Fruit and Francis Marsais}

Equipe de Chimie Organique Fine et Hétérocyclique, IRCOF-INSA

Rouen, B.P. 08, 76131 Mont-Saint-Aignan-FRANCE

laurent.bischoff@insa-rouen.fr

\section{SUPPORTING INFORMATION $-{ }^{1} \mathrm{H} \&{ }^{13} \mathrm{C}$ NMR, MS}

NMR spectra were recorded on a $300 \mathrm{MHz}$ Bruker Avance. Unless otherwise indicated, all spectra were recorded at $300 \mathrm{MHz}$ for ${ }^{1} \mathrm{H}$ and $75 \mathrm{MHz}$ for ${ }^{13} \mathrm{C}$ in $\mathrm{CDCl}_{3}$. All chemical shifts are given in ppm. $\mathrm{R} f$ given for TLC analyses were measured on silica gel Merck Kieselgel $\mathrm{F}_{254}$ aluminum plates.

\section{Standard procedure for hydroxymethylation reaction:}

To a stirred solution of n-butyllithium $(4.02 \mathrm{~mL}, 10.05 \mathrm{mmol})$ in anhydrous THF (20 mL) was added dropwise $2,2,6,6$-tetramethylpiperidine $(1.72 \mathrm{~mL}, 10.05 \mathrm{mmol})$ at $-10{ }^{\circ} \mathrm{C}$. The solution was allowed to stir at $0{ }^{\circ} \mathrm{C}$ for 30 minutes then cooled to $-78{ }^{\circ} \mathrm{C}$. Reactant $(3.35 \mathrm{mmol})$ in anhydrous THF (4 mL) was added dropwise and stirred for an additional 1 hour. $1 \mathrm{H}$ benzotriazole-methanol $(1 \mathrm{~g}, 6.70 \mathrm{mmol})$ in anhydrous THF $(30 \mathrm{~mL})$ was added dropwise over a 30 minutes period and kept 2 hours at this temperature. The reaction was quenched with water $(15 \mathrm{~mL})$ extracted with diethyl ether $(50 \mathrm{~mL})$ then washed successively with $4 \mathrm{~N}$ $\mathrm{NaOH}(15 \mathrm{~mL})$ and brine $(15 \mathrm{~mL})$. The organic layer was dried over $\mathrm{MgSO}_{4}$, filtered, and concentrated in vacuo. Purification conditions for each compound are given in this supplementary material.

Procedure is the same if $1 \mathrm{H}$-benzotriazole-methanol is replaced by $N$-hydroxymethyl phtalimide and if LTMP is replaced by LDA. 


\begin{tabular}{|c|c|}
\hline \multicolumn{2}{|l|}{ (2-bromopyridin-3-yl)methanol 4} \\
\hline$\overbrace{\mathrm{N}} \mathrm{OH}$ & $\begin{array}{l}\text { Mol. Formula : } \mathrm{C}_{6} \mathrm{H}_{6} \mathrm{BrNO} \\
\text { Mol. Weight : } 188.02\end{array}$ \\
\hline \multicolumn{2}{|c|}{$\begin{array}{l}\text { Flash chromatography eluting with dichloromethane : methanol } 95: 5(\mathrm{R} f=0.4) \text { gave a white } \\
\text { solid }(497 \mathrm{mg}, 79 \%)\end{array}$} \\
\hline \multicolumn{2}{|c|}{$\begin{array}{l}{ }^{1} \mathrm{H} \mathrm{NMR}\left(\mathrm{CDCl}_{3}, 300 \mathrm{MHz}\right): 2.23(\mathrm{t}, J=6.0 \mathrm{~Hz}, 1 \mathrm{H}), 4.75(\mathrm{~d}, J=6.0 \mathrm{~Hz}, 2 \mathrm{H}), 7.30(\mathrm{dd}, J= \\
4.7 \mathrm{~Hz}, 7.3 \mathrm{~Hz}, 1 \mathrm{H}), 7.84(\mathrm{~d}, J=7.3 \mathrm{~Hz}, 1 \mathrm{H}), 8.29(\mathrm{dd}, J=1.7 \mathrm{~Hz}, 4.7 \mathrm{~Hz}, 1 \mathrm{H})\end{array}$} \\
\hline \multicolumn{2}{|c|}{${ }^{13} \mathrm{C} \mathrm{NMR}\left(\mathrm{CDCl}_{3}, 75 \mathrm{MHz}\right): 63.4,123.2,136.7,137.5,141.6,148.7$} \\
\hline \multicolumn{2}{|c|}{ GC-MS m/z= 189, 187, 108, 106, 80, 78, 53, 51} \\
\hline Anal. calcd for $\mathrm{C}_{6} \mathrm{H}_{6} \mathrm{BrNO}: \mathrm{C}, 38 . ?$ & J, 7.45 found $\mathrm{C}, 38.28 ; \mathrm{H}, 3.17 ; \mathrm{N}, 7.51$ \\
\hline
\end{tabular}

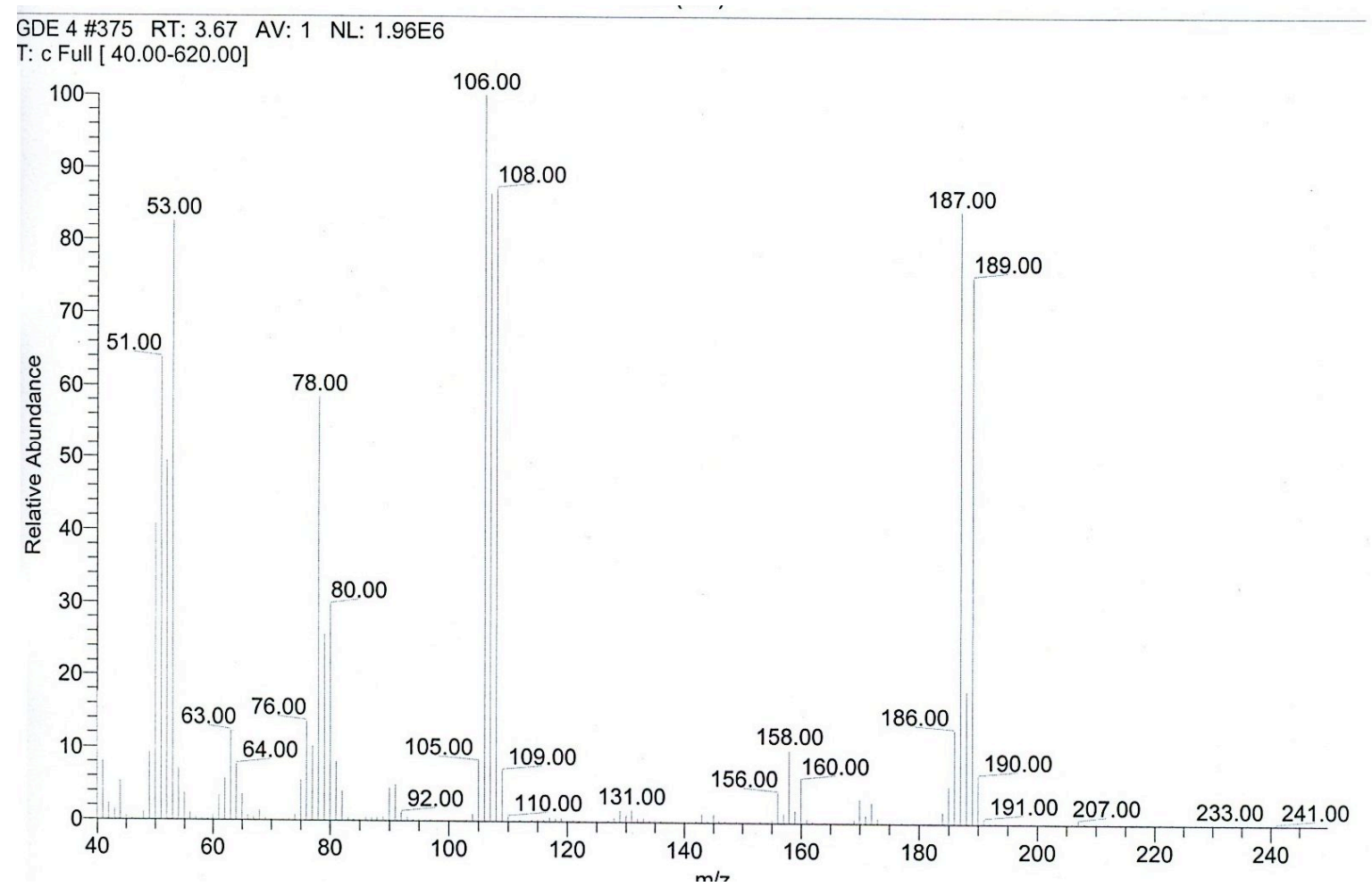


HM4-H

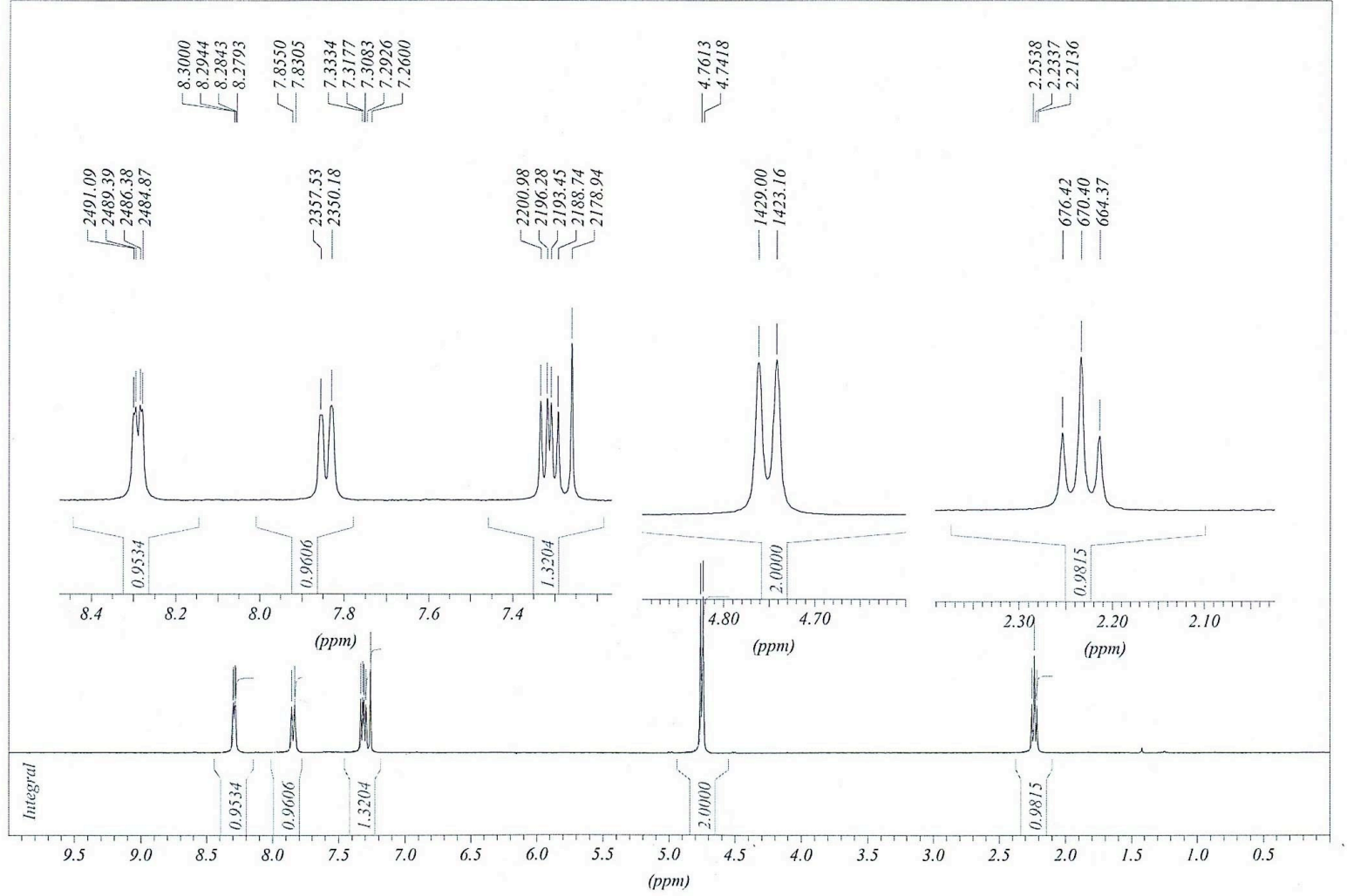

II.MAC

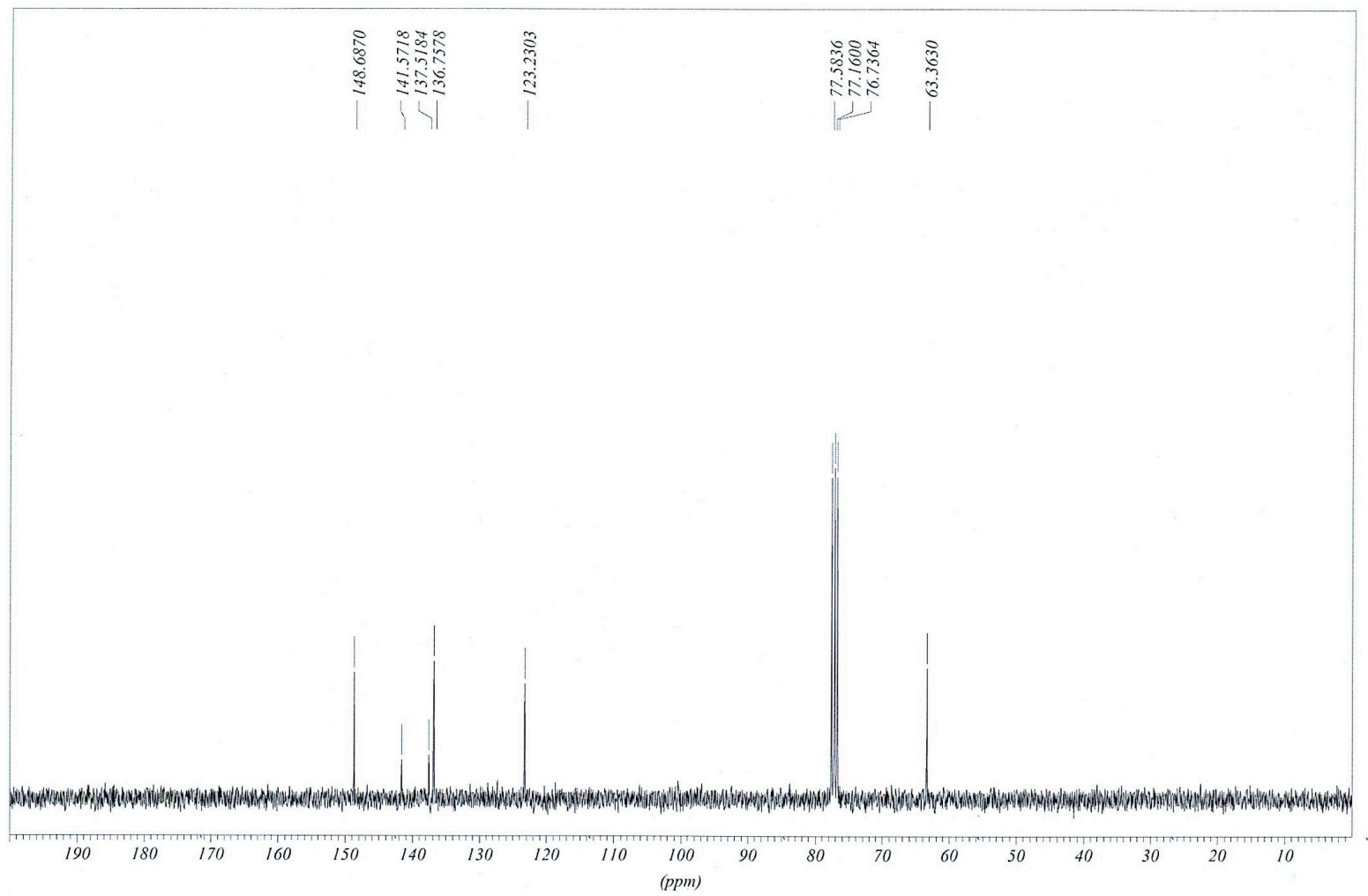


4-(hydroxymethyl)- $N, N$-diisopropylnicotinamide $\mathbf{5}$<smiles>CCCN(C(=O)c1cnccc1CO)C(C)C</smiles>

Mol. Formula : $\mathrm{C}_{13} \mathrm{H}_{20} \mathrm{~N}_{2} \mathrm{O}_{2}$

Mol. Weight : 236.31

Flash chromatography eluting with dichloromethane : methanol $95: 5(\mathrm{R} f=0.3)$, gave a yellow oil (530 mg, 67\%)

${ }^{1} \mathrm{H}$ NMR $\left(\mathrm{CDCl}_{3}, 300 \mathrm{MHz}\right): 1.01(\mathrm{~m}, 6 \mathrm{H}), 1.41(\mathrm{~d}, J=6.6 \mathrm{~Hz}, 6 \mathrm{H}), 3.45(\mathrm{~m}, 2 \mathrm{H}), 4.5(\mathrm{~m}$, $2 \mathrm{H}), 7.33(\mathrm{~d}, J=5.0 \mathrm{~Hz}, 1 \mathrm{H}), 8.19(\mathrm{~s}, 1 \mathrm{H}), 8.37(\mathrm{~d}, J=5.0 \mathrm{~Hz}, 1 \mathrm{H})$

${ }^{13} \mathrm{C}$ NMR $\left(\mathrm{CDCl}_{3}, 75 \mathrm{MHz}\right): 20.3,20.5,46.1,51.3,60.5,122.0,131.7,144.7,147.7,149.5$, 167.7

GC-MS m/z= 236, 193, 136, 86

Anal. calcd for $\mathrm{C}_{13} \mathrm{H}_{20} \mathrm{~N}_{2} \mathrm{O}_{2}$ : C, 66.07; H, 8.53; N, 11.85 found $\mathrm{C}, 66.00 ; \mathrm{H}, 8.45 ; \mathrm{N}, 11.92$

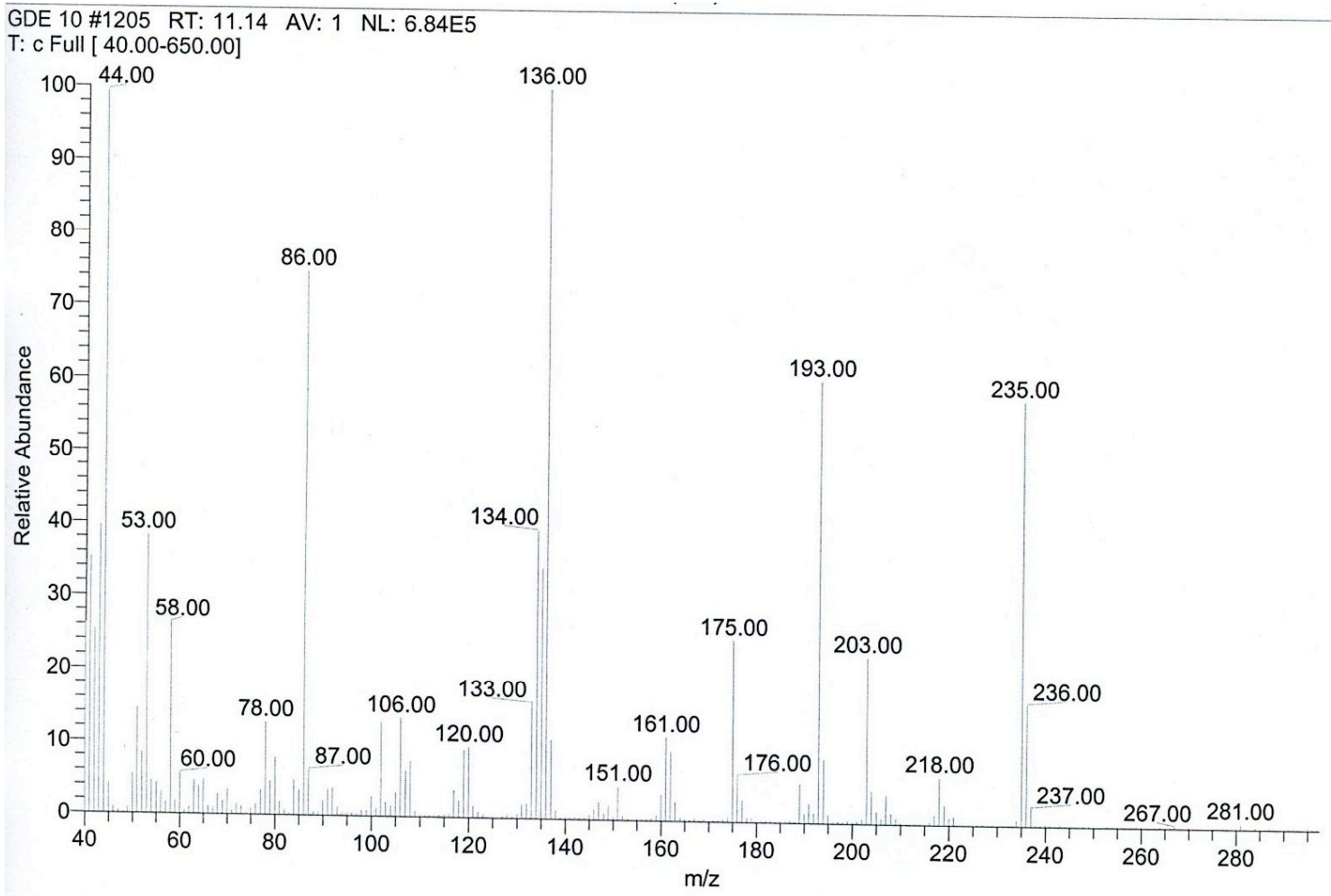




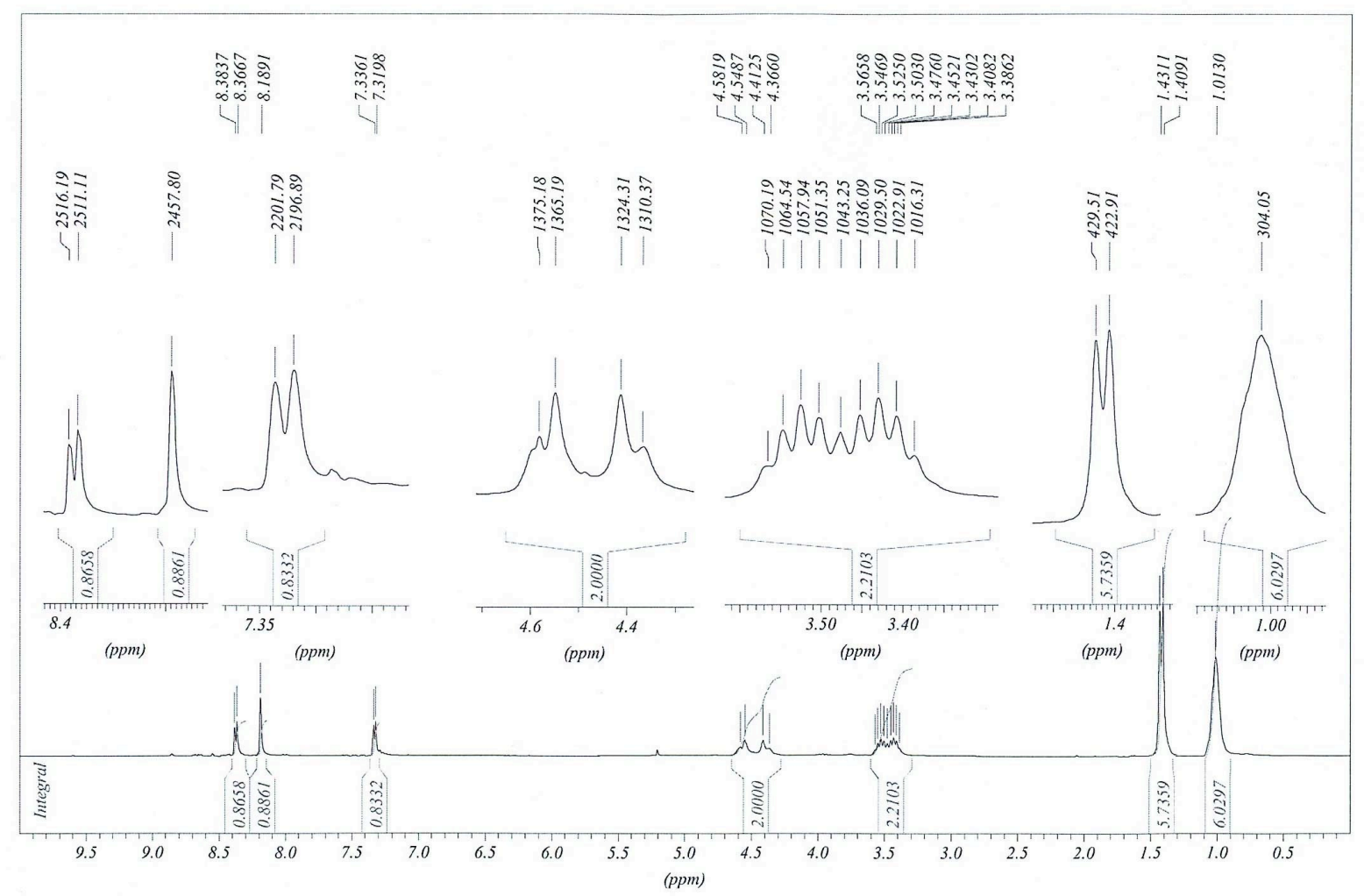

HWII) C

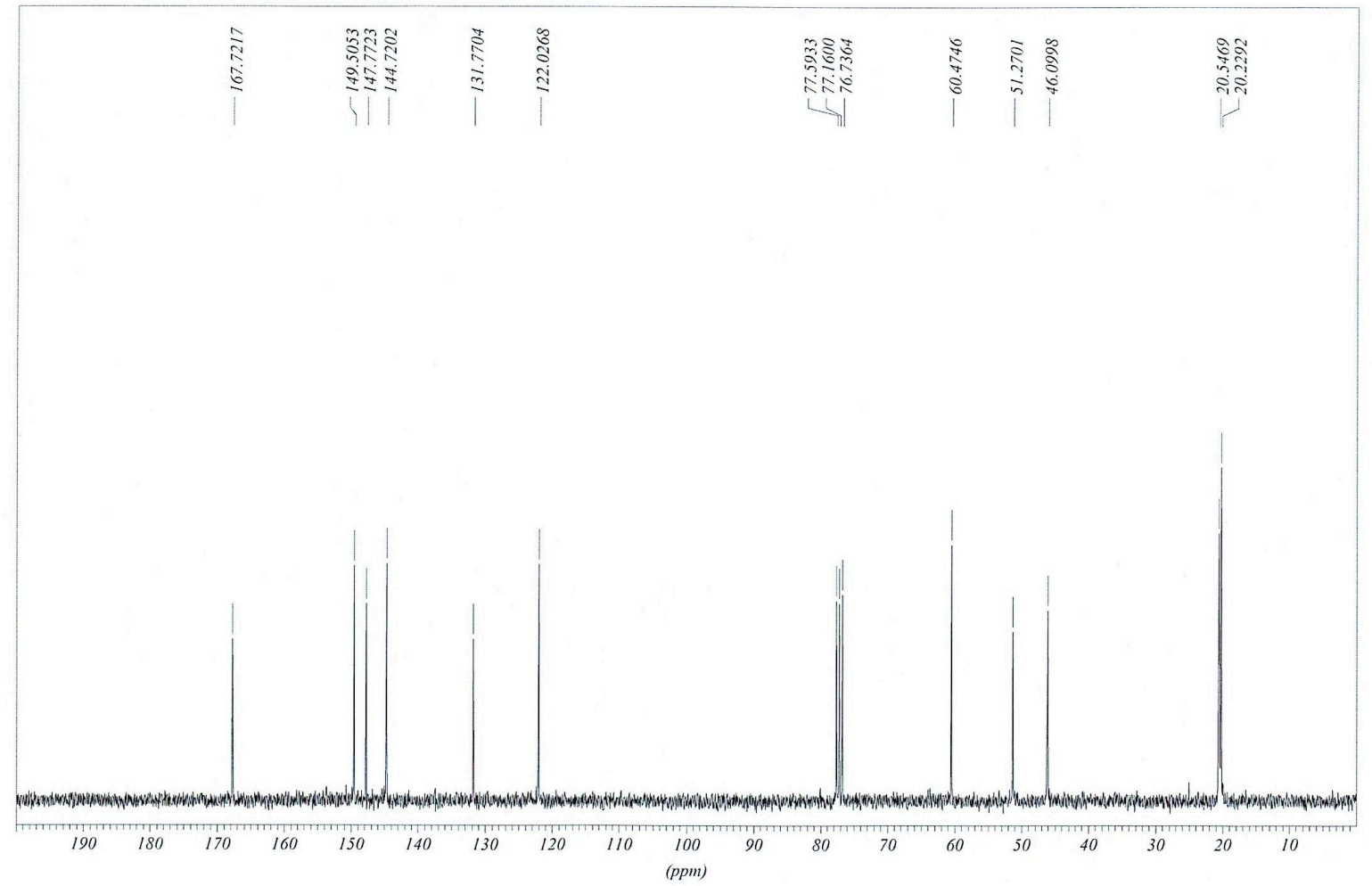


methyl 3-hydroxy-2-phenylpropanoate 6<smiles>COC(=O)C(CO)c1ccccc1</smiles>

Mol. Formula : $\mathrm{C}_{10} \mathrm{H}_{12} \mathrm{O}_{3}$

Mol. Weight : 180.20

Flash chromatography eluting with dichloromethane : methanol $95: 5(\mathrm{R} f=0.3)$, gave a colorless oil $(580 \mathrm{mg}, 96 \%)$

${ }^{1} \mathrm{H}$ NMR (CDCl 3 , $\left.300 \mathrm{MHz}\right): 3.74$ (s, 3H), 3.84 (m, 2H), 4.19 (dd, $\left.J=8.5 \mathrm{~Hz}, 10.4 \mathrm{~Hz}, 1 \mathrm{H}\right)$, $7.33(\mathrm{~m}, 5 \mathrm{H})$

${ }^{13} \mathrm{C} \mathrm{NMR}\left(\mathrm{CDCl}_{3}, 75 \mathrm{MHz}\right): 52.4,53.9,64.5,127.9,128.2,128.9,135.5,173.9$

GC-MS m/z= 180, 162, 150, 118, 91, 77, 51

Anal. calcd for $\mathrm{C}_{10} \mathrm{H}_{12} \mathrm{O}_{3}: \mathrm{C}, 66.65 ; \mathrm{H}, 6.71$ found $\mathrm{C}, 66.43 ; \mathrm{H}, 6.59$

GDE1 \#378 RT: $3.70 \quad$ AV: 1 NL: $8.38 E 6$

T: c Full [ 40.00-620.00]

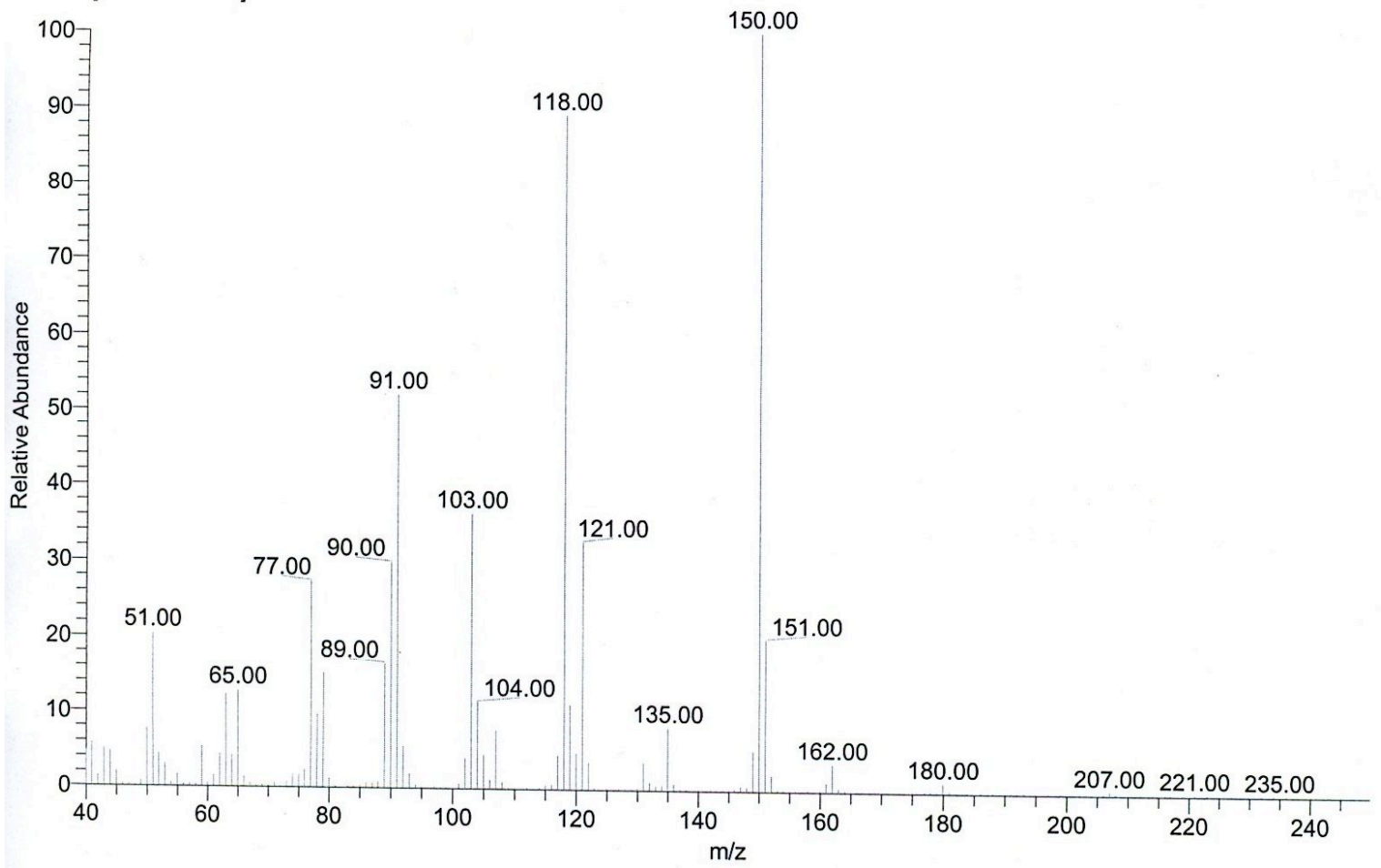




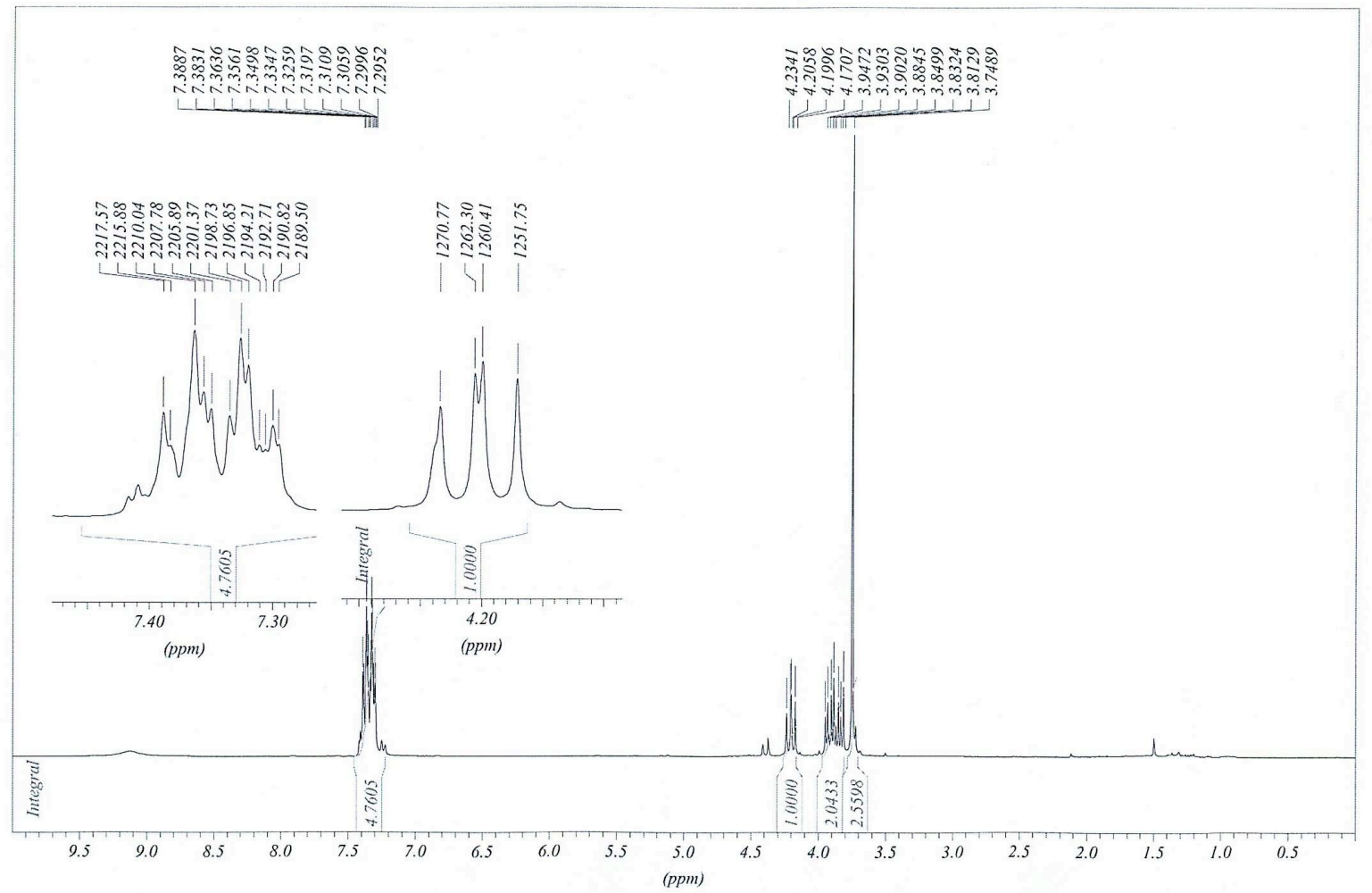

H.II-C

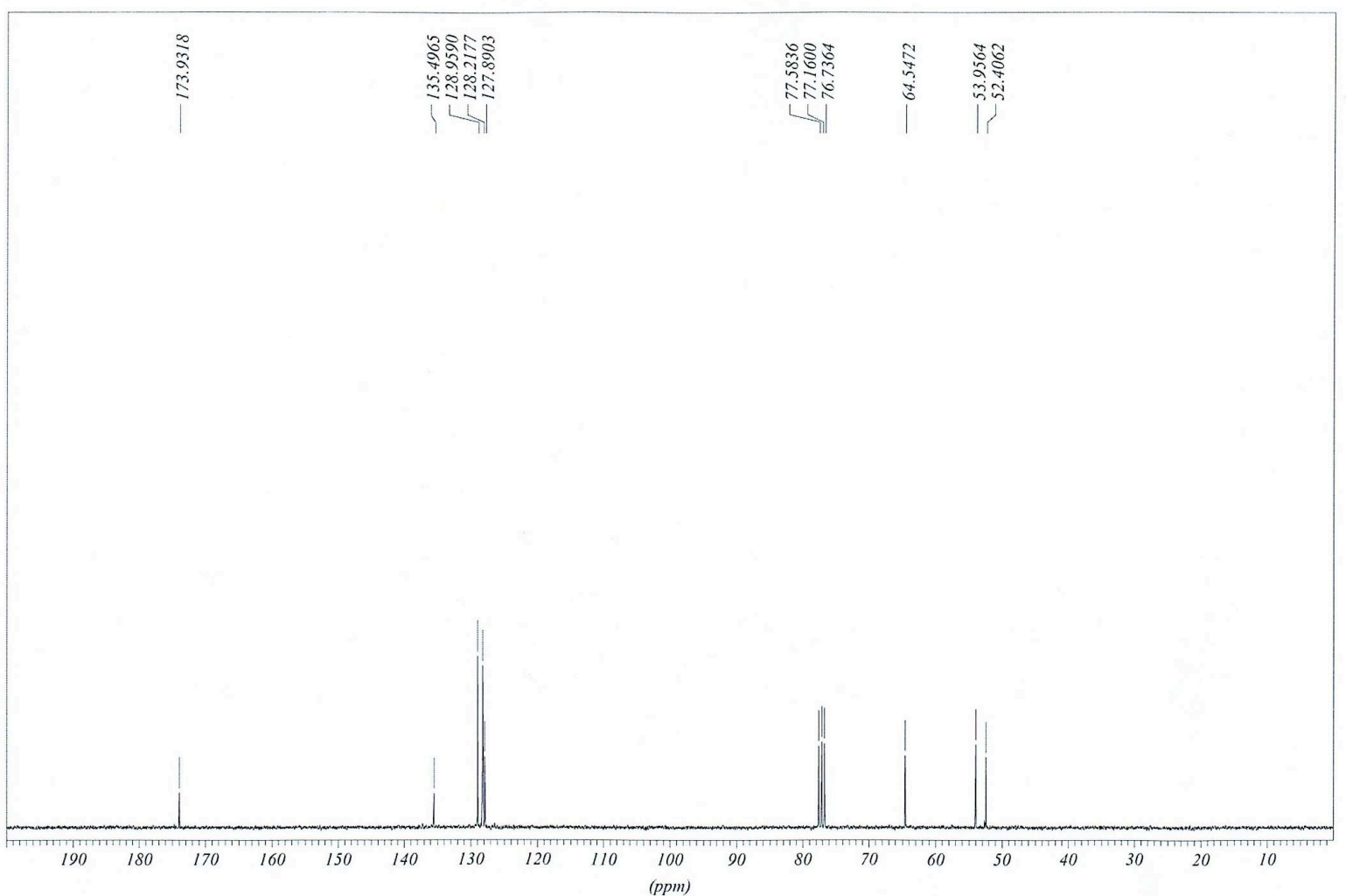


2-(hydroxymethyl)-3,4-dihydronaphthalen-1(2H)-one 7

Mol. Weight : 176.21

Flash chromatography eluting with ethyl acetate : cyclohexane $1: 1(\mathrm{R} f=0.5)$, gave a colorless oil (372 $\mathrm{mg}, 63 \%)$

${ }^{1} \mathrm{H}$ NMR $\left(\mathrm{CDCl}_{3}, 300 \mathrm{MHz}\right): 1.90(\mathrm{ddd}, J=5.1 \mathrm{~Hz}, 13.3 \mathrm{~Hz}, 18.4 \mathrm{~Hz}, 1 \mathrm{H}), 2.11(\mathrm{~m}, 1 \mathrm{H}), 2.72$ $(\mathrm{m}, 1 \mathrm{H}), 3.1(\mathrm{~m}, 3 \mathrm{H}), 3.87(\mathrm{ddd}, J=7.3 \mathrm{~Hz}, 11.3 \mathrm{~Hz}, 18.4 \mathrm{~Hz}, 2 \mathrm{H}), 7.27(\mathrm{~m}, 2 \mathrm{H}), 7.50(\mathrm{td}, J=$ $1.3 \mathrm{~Hz}, 7.7 \mathrm{~Hz}, 1 \mathrm{H}), 8.01(\mathrm{~d}, J=7.7 \mathrm{~Hz}, 1 \mathrm{H})$

${ }^{13} \mathrm{C} \mathrm{NMR}\left(\mathrm{CDCl}_{3}, 75 \mathrm{MHz}\right): 26.3,29.1,49.5,63.8,126.8,127.3,128.9,132.4,133.9,144.4$, 201.8

GC-MS m/z= 176, 158, 130, 118, 90

Anal. calcd for $\mathrm{C}_{11} \mathrm{H}_{12} \mathrm{O}_{2}: \mathrm{C}, 74.98 ; \mathrm{H}, 6.86$ found $\mathrm{C}, 74.84 ; \mathrm{H}, 6.75$

GDE2 \#896-900 RT: 7.45-7.48 AV: 5 SB: 21 7.32-7.38, 7.67-7.77 NL: 2.44E5

T: c Full [ 20.00-500.00]

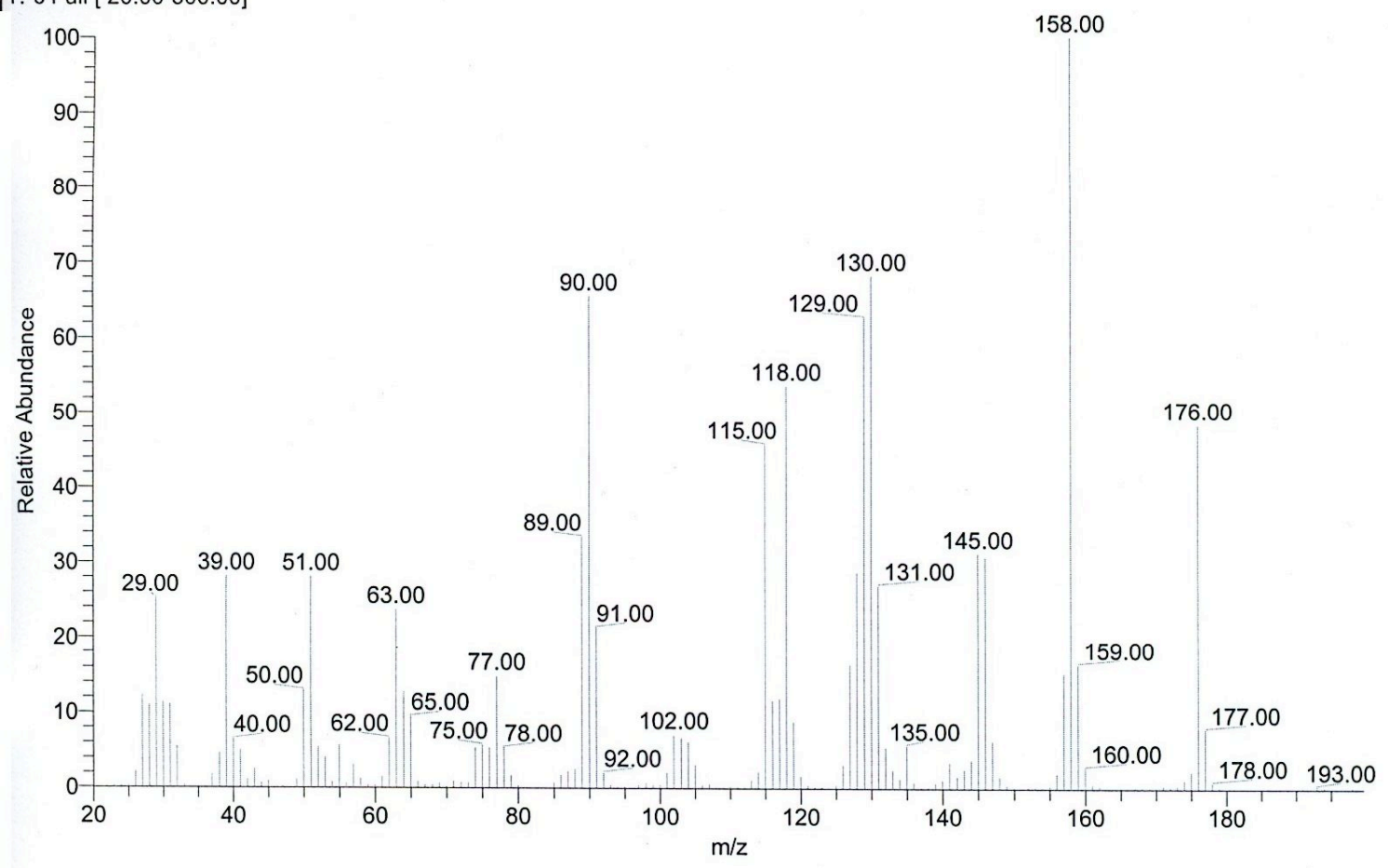




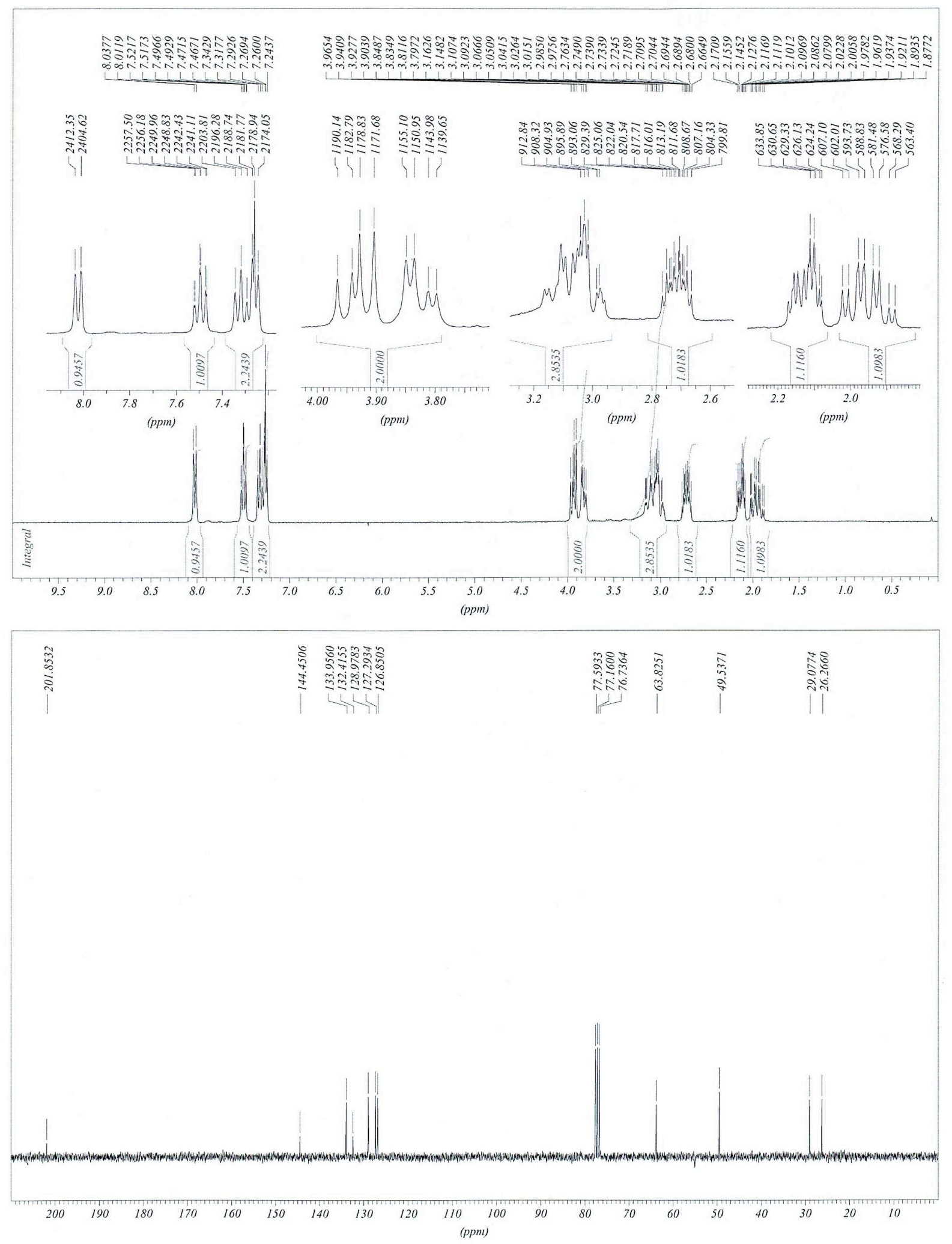


2,2-bis(hydroxymethyl)-3,4-dihydronaphthalen-1(2H)-one $\mathbf{8}$<smiles>O=C1c2ccccc2CCC1(CO)CO</smiles>
Mol. Formula : $\mathrm{C}_{12} \mathrm{H}_{14} \mathrm{O}_{3}$

Mol. Weight : 206.24

Deprotonation was accomplished with commercially available potassium tert-butoxide at $-78^{\circ} \mathrm{C}$. Flash chromatography eluting with ethyl acetate : cyclohexane $1: 1(\mathrm{R} f=0.25)$, gave a colorless oil $(636 \mathrm{mg}, 92 \%)$

${ }^{1} \mathrm{H} \mathrm{NMR}\left(\mathrm{CDCl}_{3}, 300 \mathrm{MHz}\right): 1.93(\mathrm{t}, J=6.5 \mathrm{~Hz}, 2 \mathrm{H}), 2.97(\mathrm{t}, J=6.5 \mathrm{~Hz}, 2 \mathrm{H}), 3.11(\mathrm{bs}, 1 \mathrm{H})$, $3.69(\mathrm{~d}, J=11.3 \mathrm{~Hz}, 2 \mathrm{H}), 3.90(\mathrm{~d}, J=11.3 \mathrm{~Hz}, 2 \mathrm{H}), 7.19(\mathrm{~m}, 2 \mathrm{H}), 7.45(\mathrm{td}, \mathrm{J}=1.2 \mathrm{~Hz}, 7.7$ $\mathrm{Hz}, 1 \mathrm{H}), 7.94(\mathrm{~d}, J=7.7 \mathrm{~Hz}, 1 \mathrm{H})$

${ }^{13} \mathrm{C} \mathrm{NMR}\left(\mathrm{CDCl}_{3}, 75 \mathrm{MHz}\right): 24.9,26.5,50.9,64.9,127.0,127.7,129.0,131.7,134.3,143.7$, 203.4

$\mathrm{IC}(+)-\mathrm{MS} \mathrm{MH}=207$

Anal. calcd for $\mathrm{C}_{6} \mathrm{H}_{7} \mathrm{NO}$ : C, 69.88; $\mathrm{H}, 6.84$ found $\mathrm{C}, 69.72 ; \mathrm{H}, 6.75$

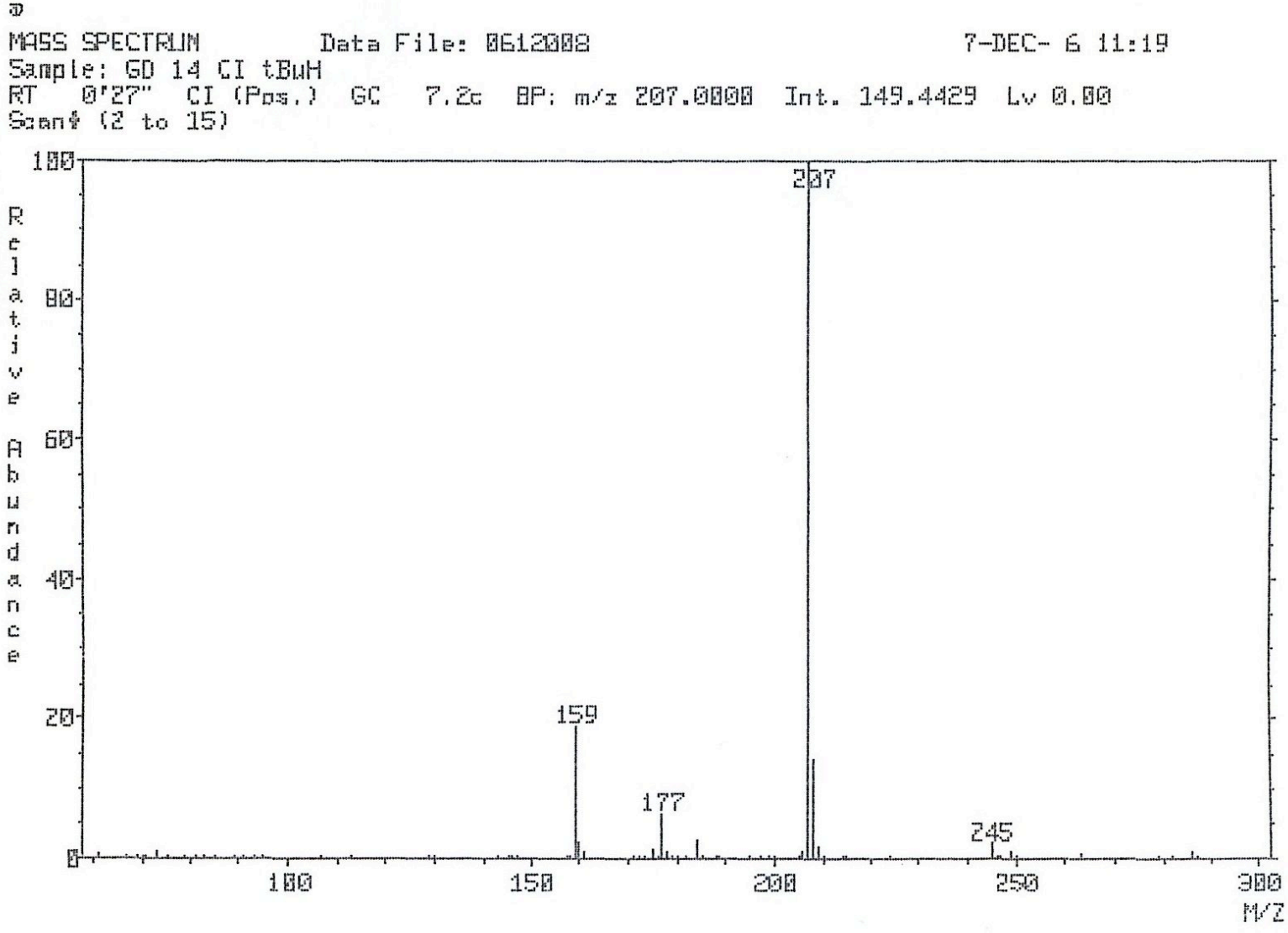




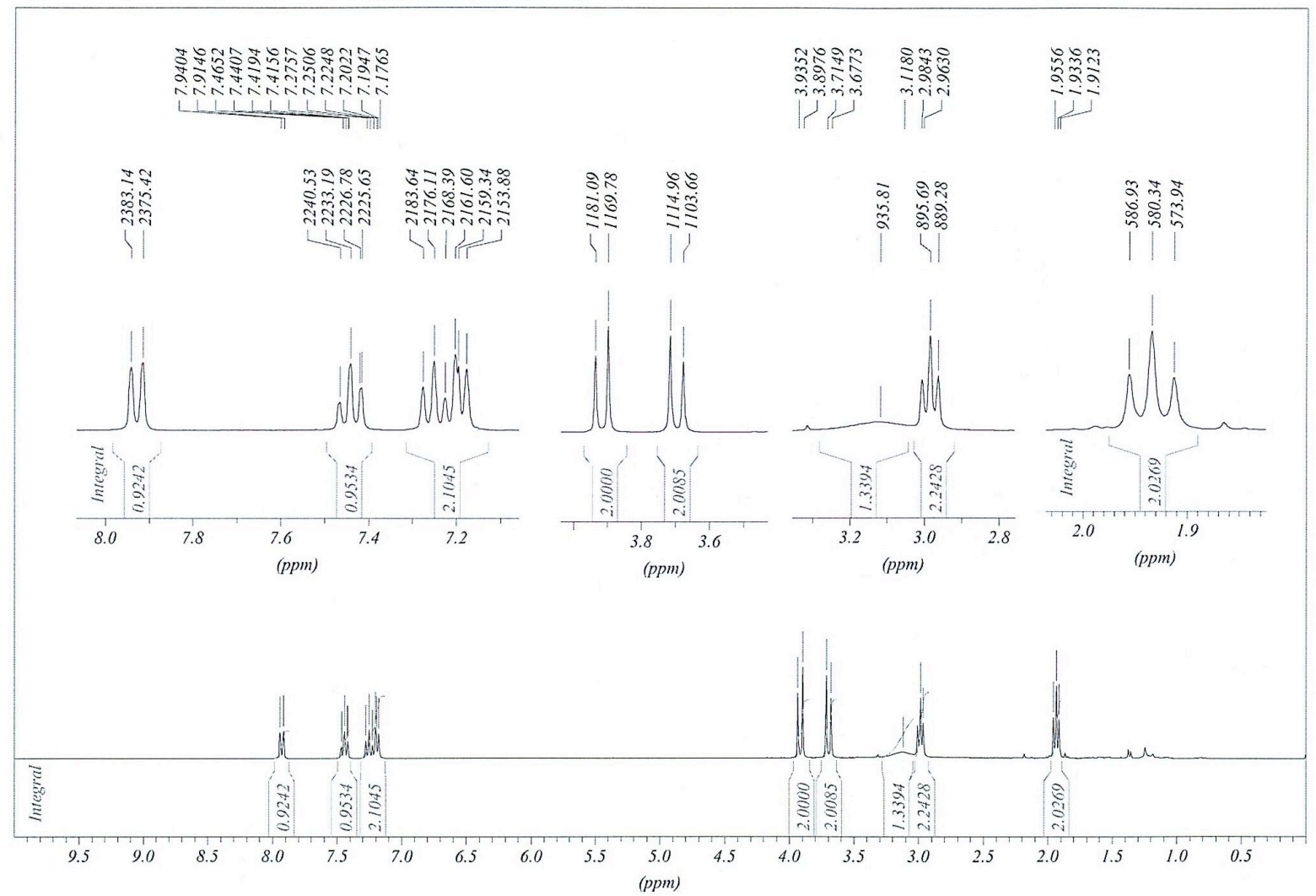

Hawe $x$

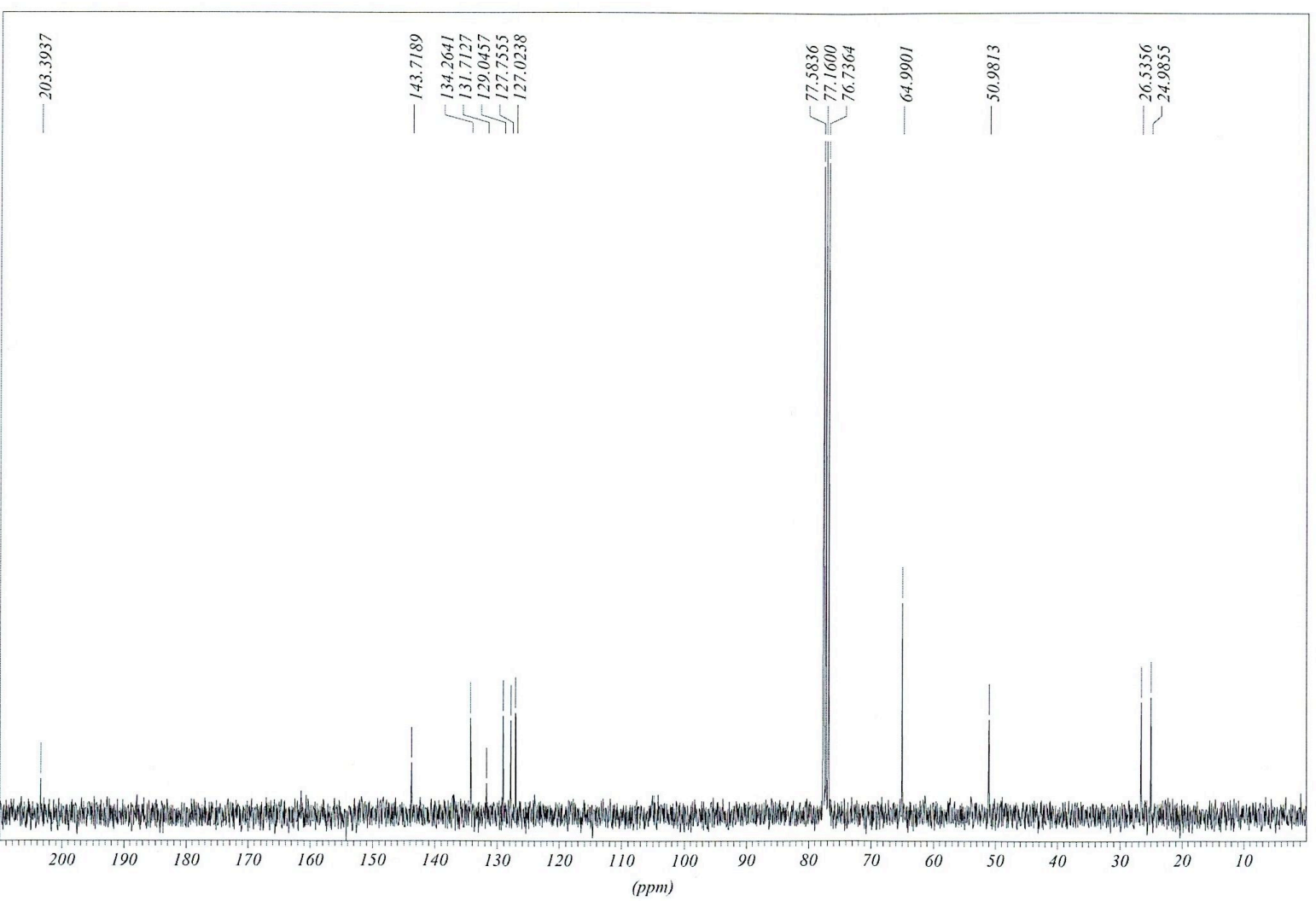


2-(hydroxymethyl)-2-methyl-3,4-dihydronaphthalen-1(2H)-one 9

Mol. Weight : 190.24

Flash chromatography eluting with dichloromethane : methanol $95: 5(\mathrm{R} f=0.6)$, gave a yellow oil (618 mg, 97\%)

${ }^{1} \mathrm{H} \mathrm{NMR}\left(\mathrm{CDCl}_{3}, 300 \mathrm{MHz}\right): 1.14(\mathrm{~s}, 3 \mathrm{H}), 1.71(\mathrm{td}, J=4.5 \mathrm{~Hz}, 8.5 \mathrm{~Hz}, 1 \mathrm{H}), 2.17(\mathrm{td}, J=5.1$ $\mathrm{Hz}, 13.2 \mathrm{~Hz}, 1 \mathrm{H}), 2.83(\mathrm{td}, J=4.0 \mathrm{~Hz}, 4.4 \mathrm{~Hz}, 2 \mathrm{H}), 3.05(\mathrm{~m}, 1 \mathrm{H}), 3.60(\mathrm{dd}, J=11.1 \mathrm{~Hz}, 32.0$ $\mathrm{Hz}, 2 \mathrm{H}), 7.19(\mathrm{~m}, 2 \mathrm{H}), 7.40(\mathrm{t}, J=7.3 \mathrm{~Hz}, 1 \mathrm{H}), 7.94(\mathrm{~d}, J=7.9 \mathrm{~Hz}, 1 \mathrm{H})$

${ }^{13} \mathrm{C} \mathrm{NMR}\left(\mathrm{CDCl}_{3}, 75 \mathrm{MHz}\right): 17.7,24.3,30.5,45.7,67.9,125.9,126.7,128.0,130.6,132.8$, $142.7,202.9$

GC-MS m/z= 190, 172, 160, 145, 118, 90

Anal. calcd for $\mathrm{C}_{12} \mathrm{H}_{14} \mathrm{O}_{2}$ : C, 75.76; $\mathrm{H}, 6.86$ found $\mathrm{C}, 75.66 ; \mathrm{H}, 6.81$

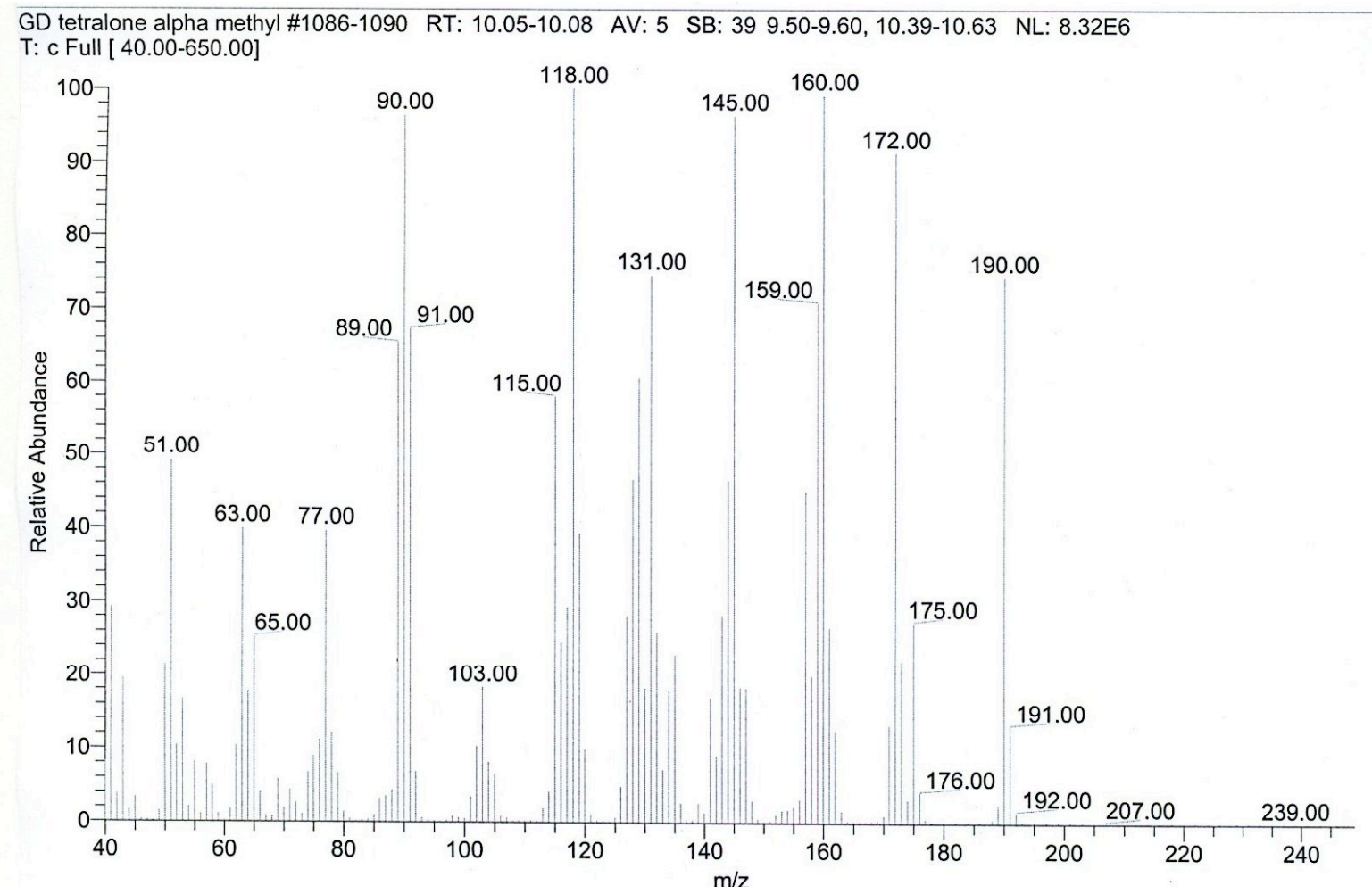




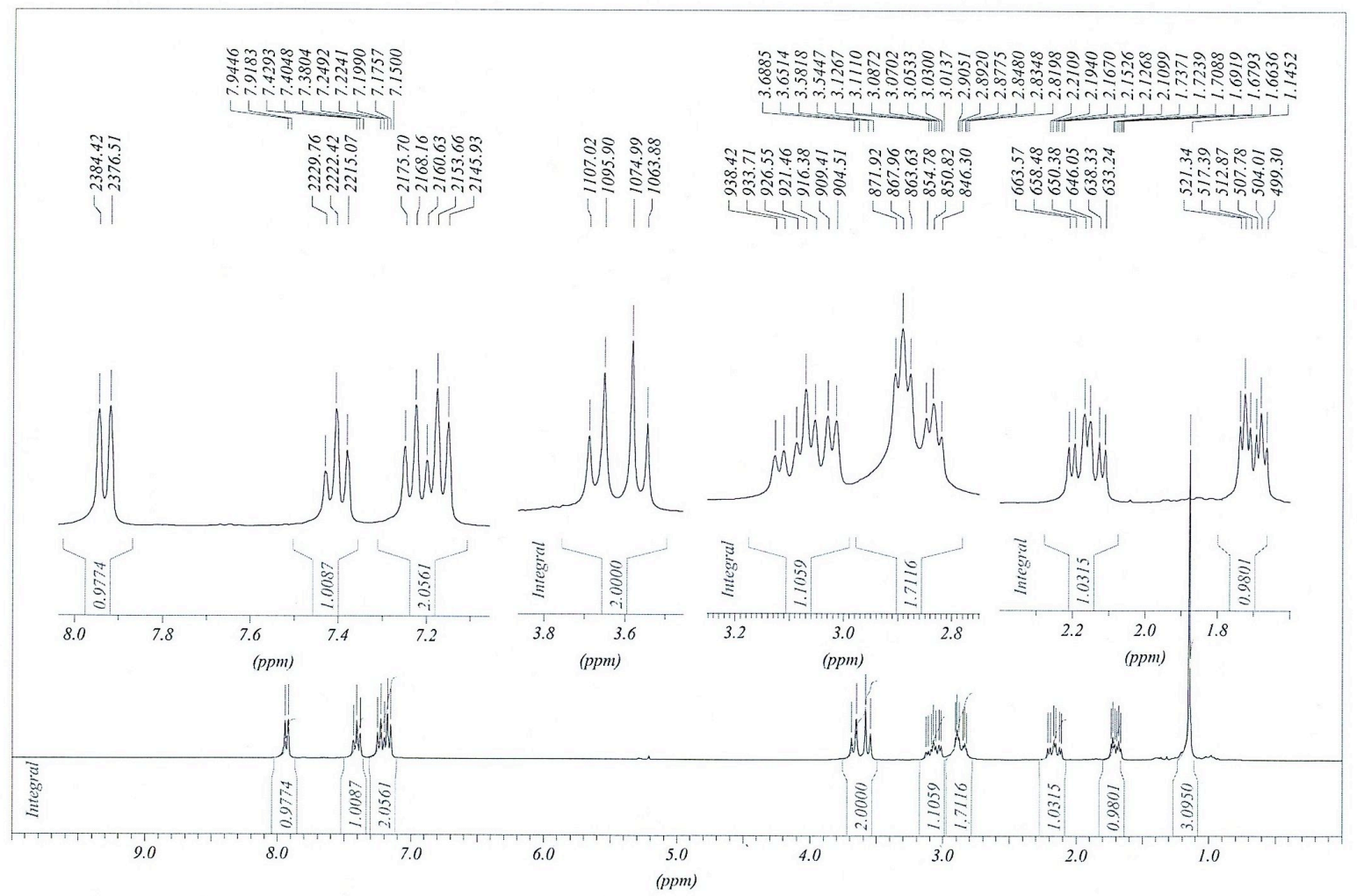

$H A B-C$

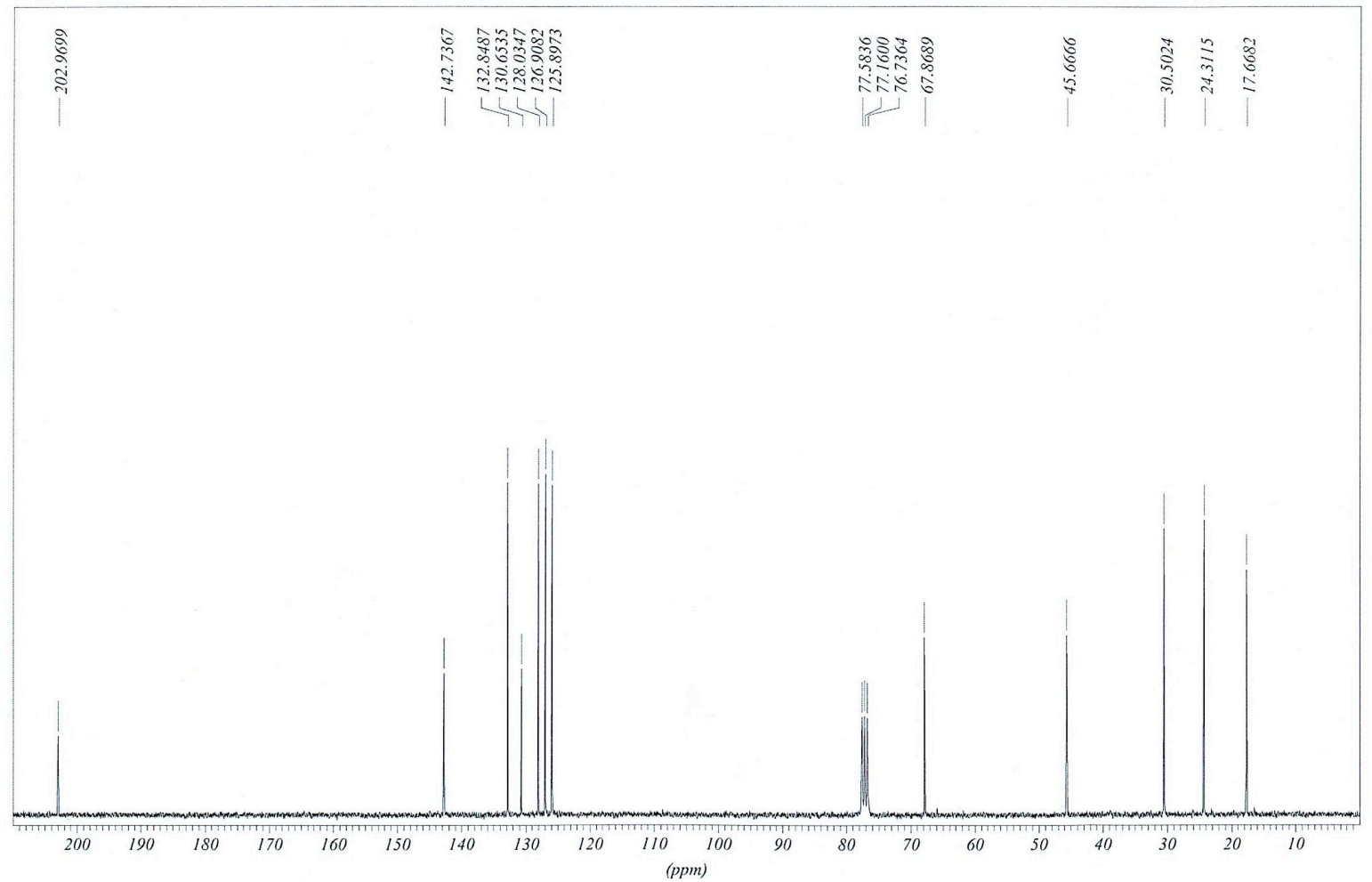


3-hydroxy-2-methylpropanenitrile $\mathbf{1 0}$

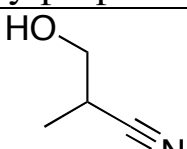

Mol. Formula : $\mathrm{C}_{4} \mathrm{H}_{7} \mathrm{NO}$

Mol. Weight : 85.1

Evaporation of the solvents and starting material gave a colorless oil (192 mg, 67\%)

${ }^{1} \mathrm{H} \mathrm{NMR}\left(\mathrm{CDCl}_{3}, 300 \mathrm{MHz}\right): 1.29(\mathrm{~d}, J=7.2 \mathrm{~Hz}, 3 \mathrm{H}), 2.80(\mathrm{~m}, 1 \mathrm{H}), 2.95(\mathrm{~m}, 1 \mathrm{H}), 3.71(\mathrm{~d}, J=$ $6.2 \mathrm{~Hz}, 1 \mathrm{H}), 3.71(\mathrm{~d}, J=6.2 \mathrm{~Hz}, 1 \mathrm{H})$

${ }^{13} \mathrm{C} \mathrm{NMR}\left(\mathrm{CDCl}_{3}, 75 \mathrm{MHz}\right): 14.2,29.0,63.8,121.8$

GC-MS $\mathrm{m} / \mathrm{z}=$ not detected

Anal. calcd for $\mathrm{C}_{4} \mathrm{H}_{7} \mathrm{NO}$ : C, 56.45; H, 8.29; N, 16.46 found $\mathrm{C}, 56.37 ; \mathrm{H}, 8.22 ; \mathrm{N}, 16.55$ 


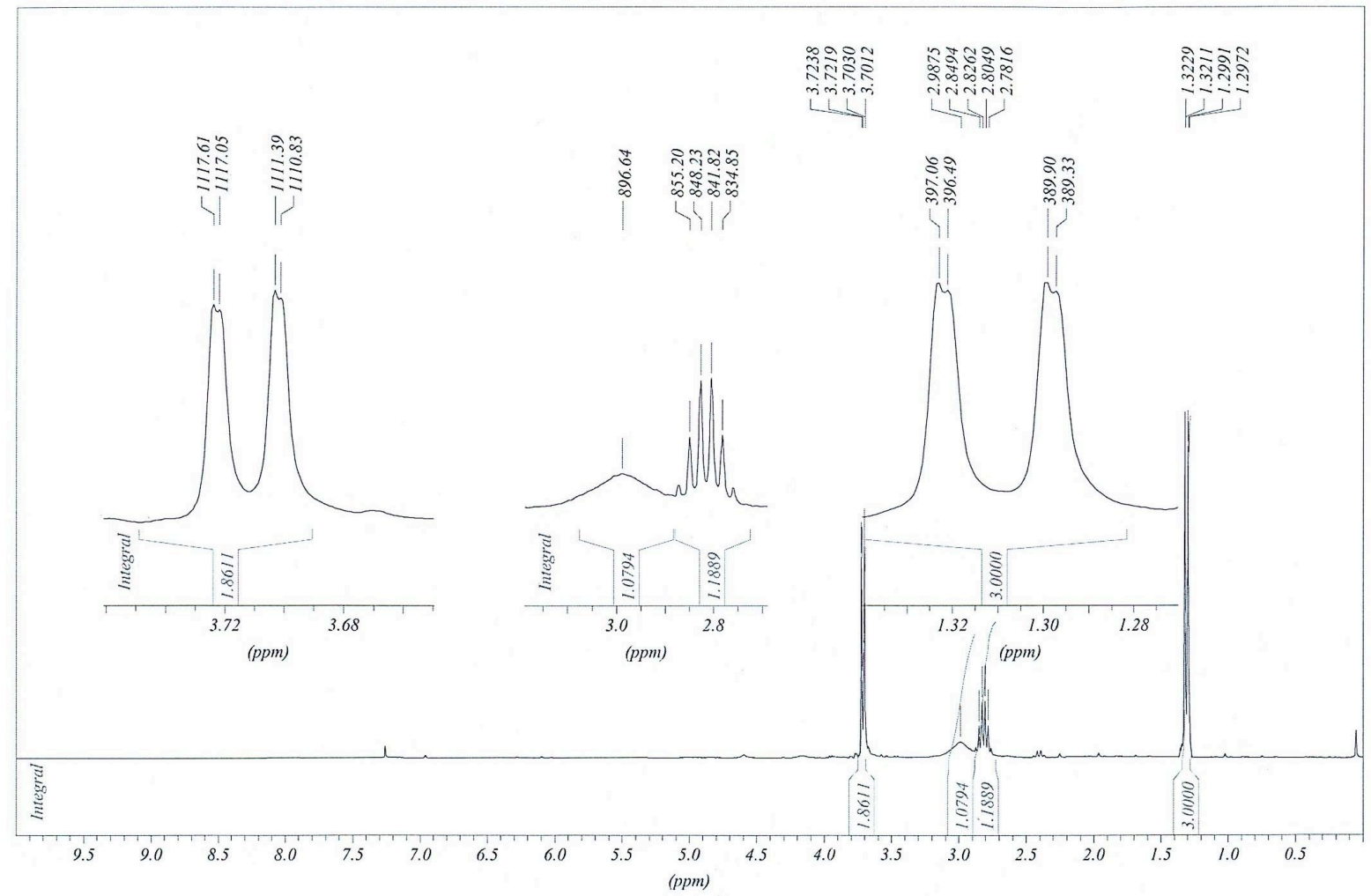

H:H2-C

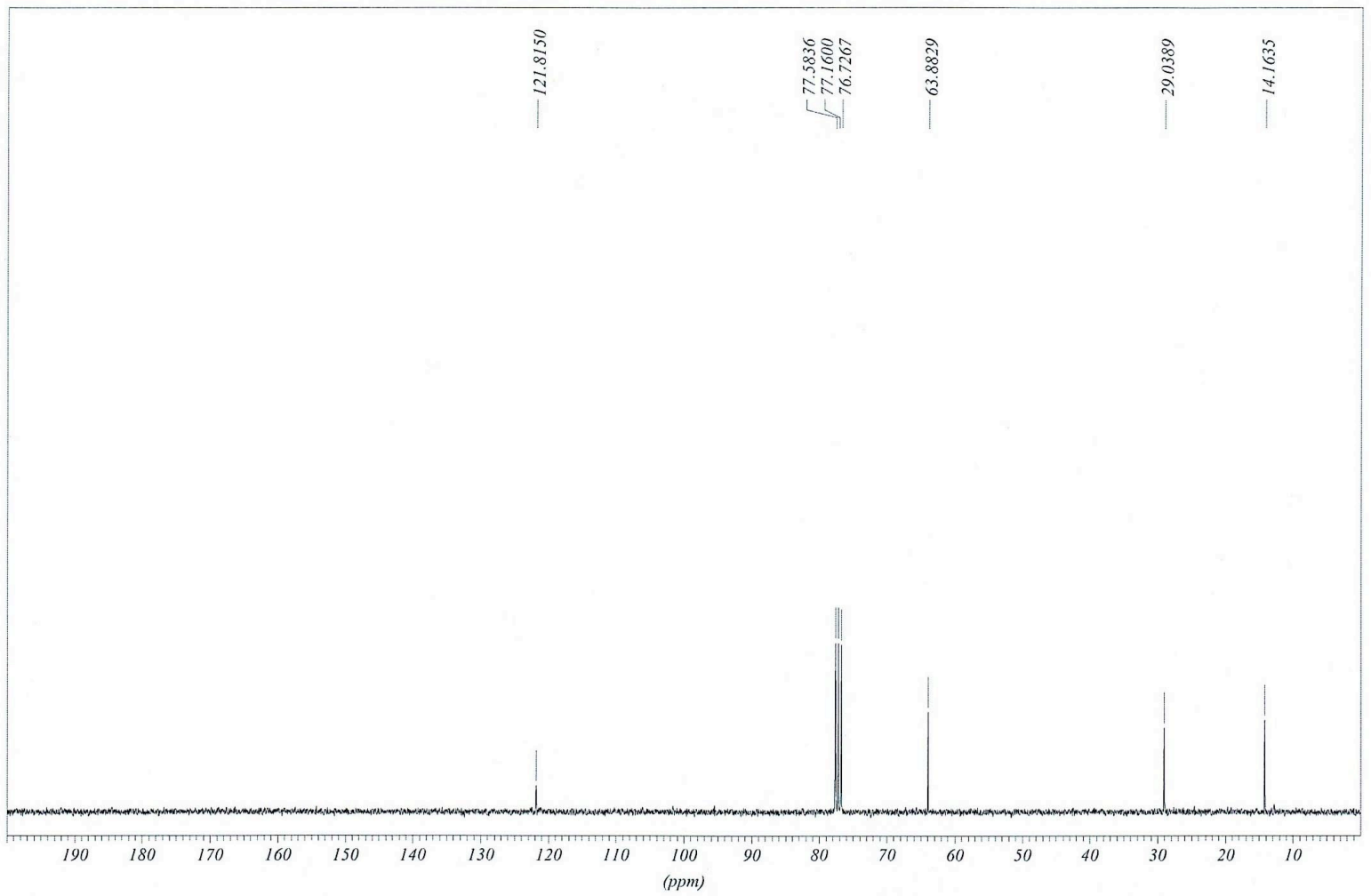


<smiles>O=S(=O)(CCO)c1ccccc1</smiles>

Mol. Weight : 186.23

Flash chromatography eluting with dichloromethane : methanol $95: 5(\mathrm{R} f=0.45)$, gave a colorless oil (330 mg, 53\%)

${ }^{1} \mathrm{H} \mathrm{NMR}\left(\mathrm{CDCl}_{3}, 300 \mathrm{MHz}\right): 3.29(\mathrm{t}, J=5.6 \mathrm{~Hz}, 2 \mathrm{H}), 3.90(\mathrm{t}, J=5.6 \mathrm{~Hz}, 2 \mathrm{H}), 7.50(\mathrm{~m}, 2 \mathrm{H})$, $7.57(\mathrm{~m}, 1 \mathrm{H}), 7.84(\mathrm{~m}, 2 \mathrm{H})$

${ }^{13} \mathrm{C} \mathrm{NMR}\left(\mathrm{CDCl}_{3}, 75 \mathrm{MHz}\right): 56.2,58.2,127.9,129.5,134.1,138.9$

GC-MS m/z= 186, 158, 143, 125, 77

Anal. calcd for $\mathrm{C}_{8} \mathrm{H}_{10} \mathrm{O}_{3} \mathrm{~S}: \mathrm{C}, 51.60 ; \mathrm{H}, 5.41 ; \mathrm{S}, 17.22$ found $\mathrm{C}, 51.55 ; \mathrm{H}, 5.37 ; \mathrm{S}, 17.15$

GDE8 \#1473-1487 RT: 12.22-12.34 AV: 15 SB: 81 11.76-11.97, 13.07-13.52 NL: 2.39E6

T: c Full [ 20.00-500.00]

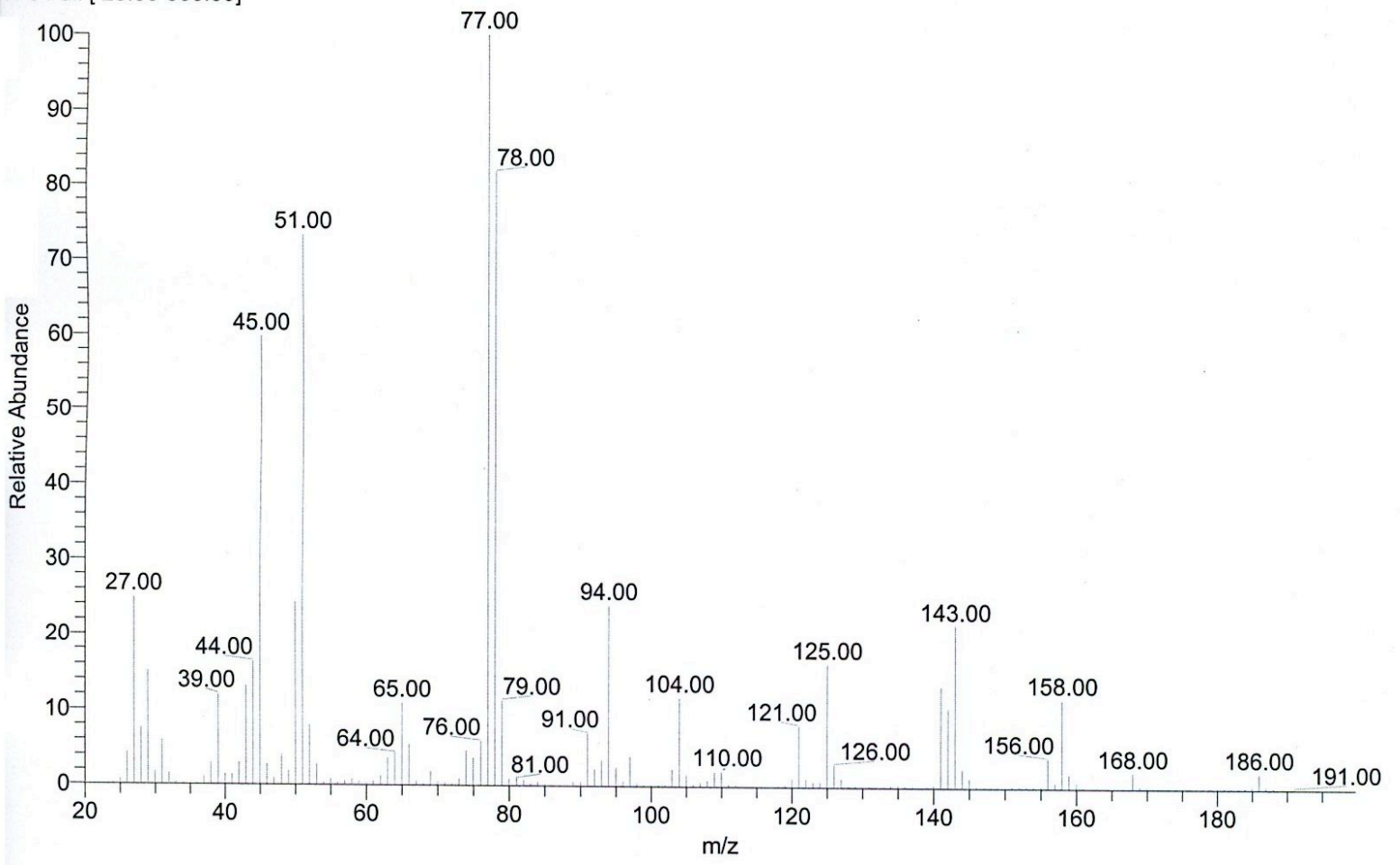




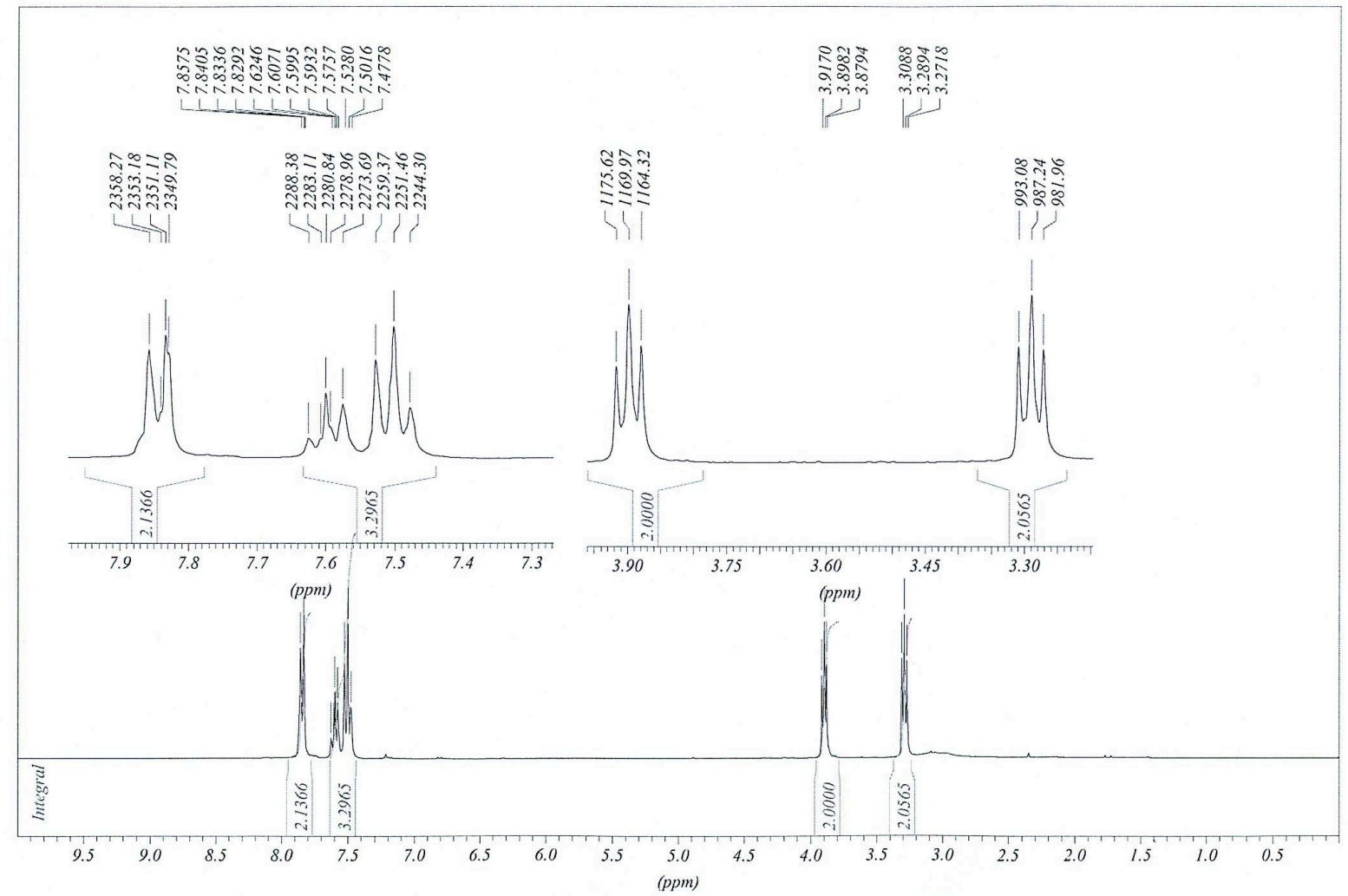

HWIS-C
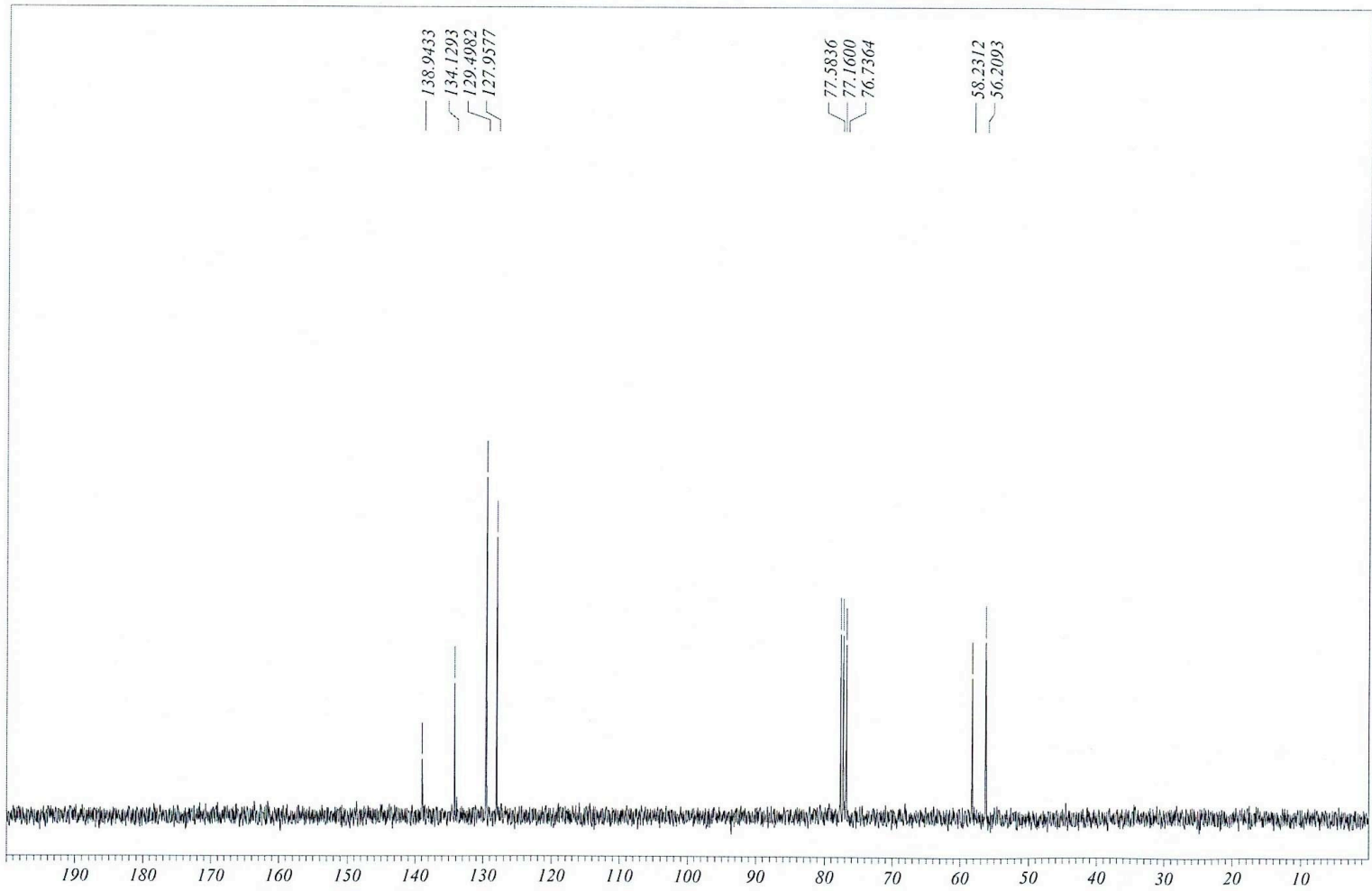


\begin{tabular}{|l|l|}
\hline 2-(pyridin-2-yl)ethanol 12 & Mol. Formula $: \mathrm{C}_{7} \mathrm{H}_{9} \mathrm{NO}$ \\
\hline Evaporation of the solvents and starting material gave a colorless oil $(268 \mathrm{mg}, 65 \%)$ \\
\hline${ }^{1} \mathrm{H} ~ \mathrm{NMR}\left(\mathrm{CDCl}_{3}, 300 \mathrm{MHz}\right): 2.98(\mathrm{t}, \mathrm{J}=5.5 \mathrm{~Hz}, 1 \mathrm{H}), 2.99(\mathrm{t}, J=5.6 \mathrm{~Hz}, 1 \mathrm{H}), 3.98(\mathrm{t}, J=5.6$ \\
$\mathrm{Hz}, 1 \mathrm{H}), 3.99(\mathrm{t}, J=5.5 \mathrm{~Hz}, 1 \mathrm{H}), 7.12(\mathrm{~m}, 2 \mathrm{H}), 7.61(\mathrm{~m}, 1 \mathrm{H}), 8.44(\mathrm{~m}, 1 \mathrm{H})$
\end{tabular}

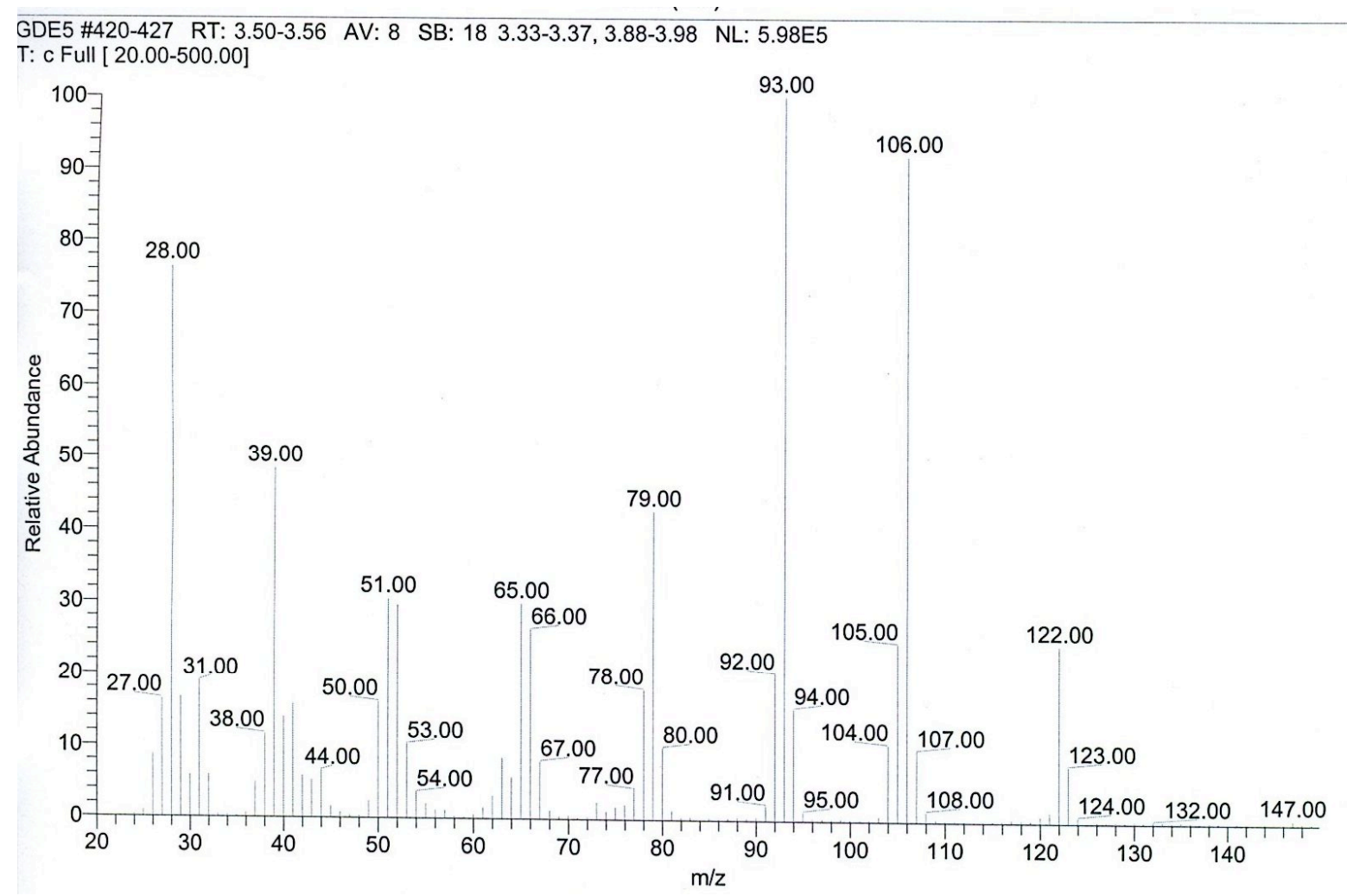




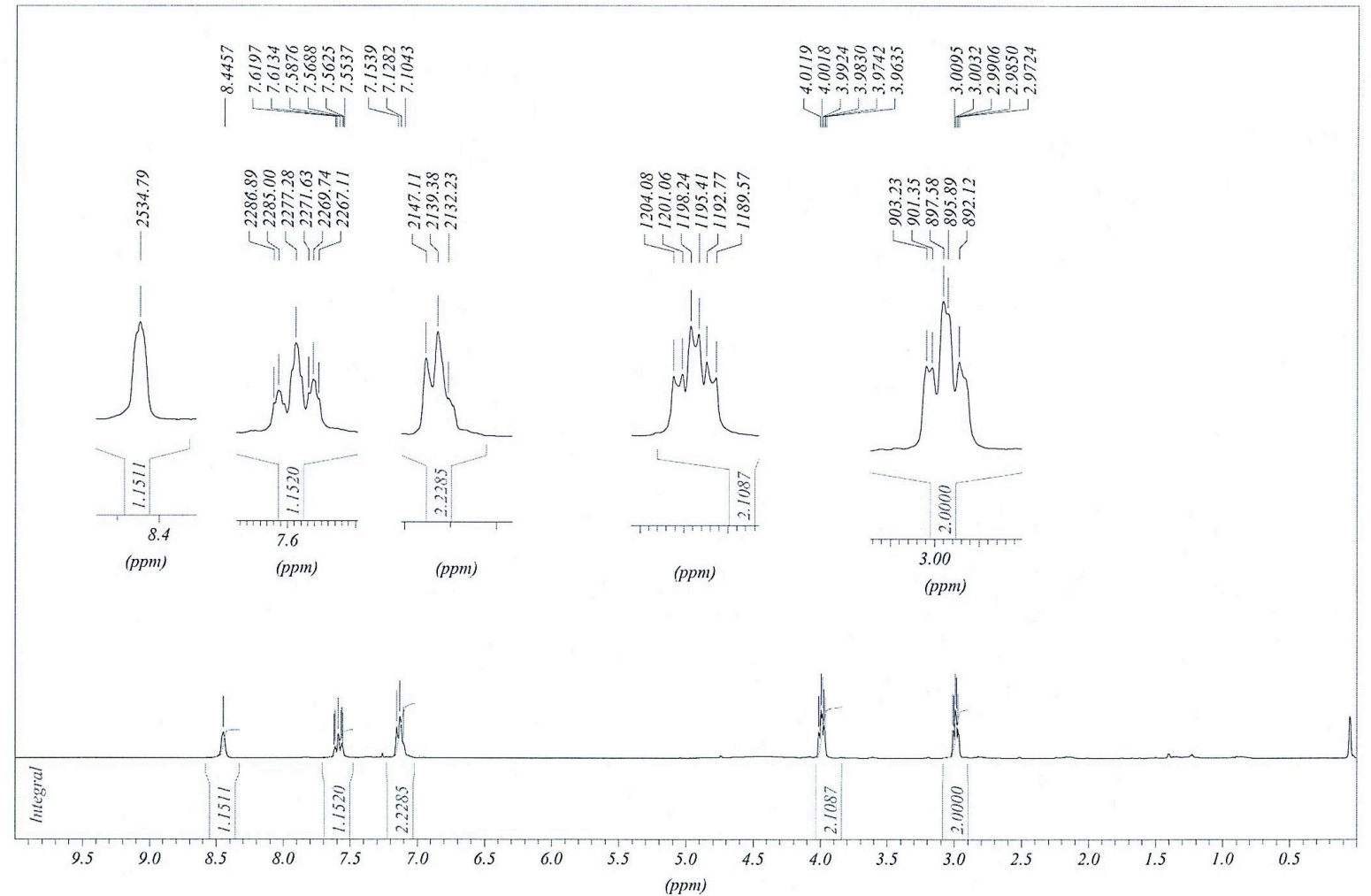

MISE-C

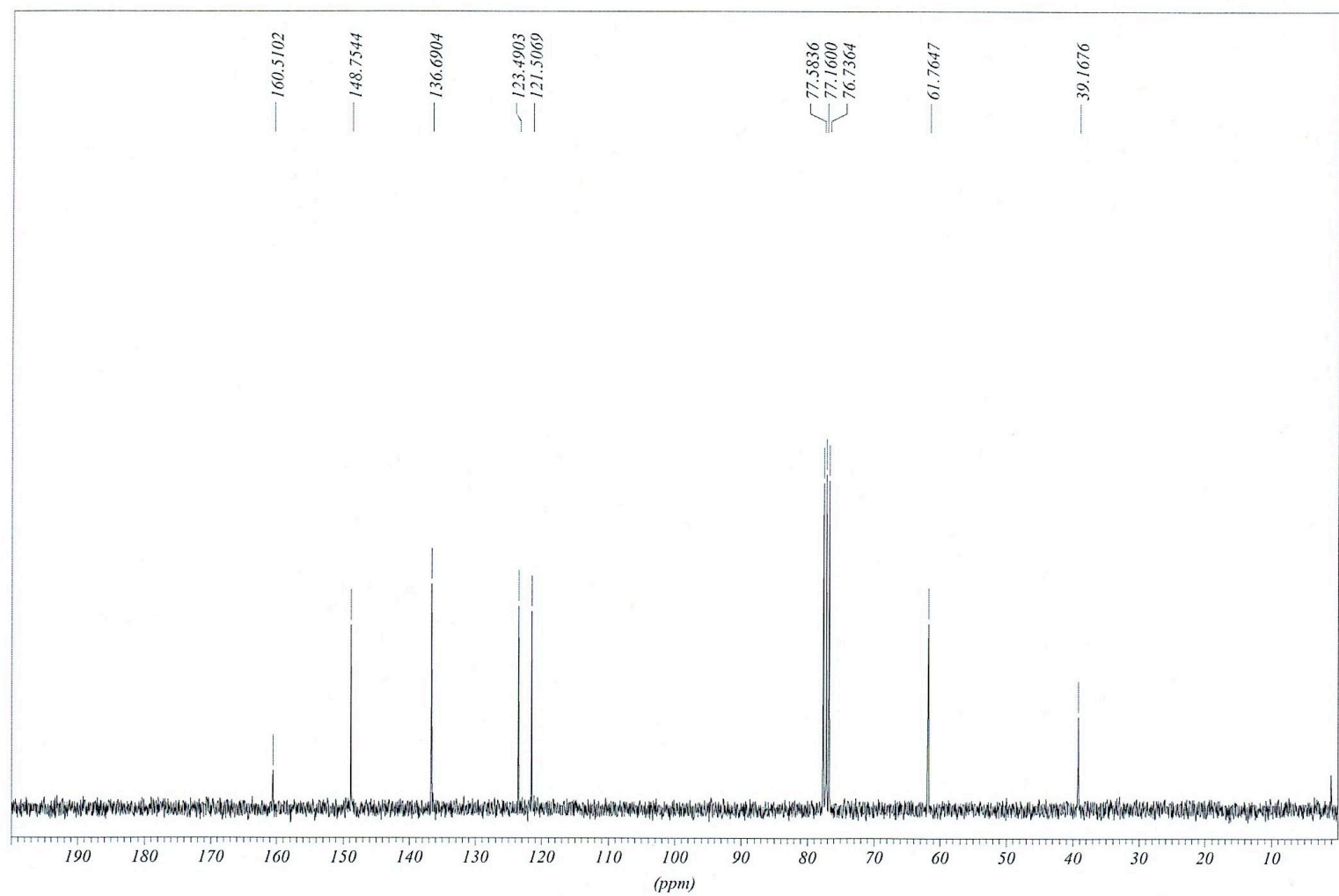




\begin{tabular}{|l|l|}
\hline 3-phenylprop-2-yn-1-ol 13 & Mol. Formula $: \mathrm{C}_{9} \mathrm{H}_{8} \mathrm{O}$ \\
\hline $\begin{array}{l}\text { Flash chromatography eluting with dichloromethane : methanol } 95: 5(\mathrm{Rf}=0.6) \text {, gave a } \\
\text { colorless oil }(226 \mathrm{mg}, 51 \%)\end{array}$ \\
\hline${ }^{1} \mathrm{H}$ NMR $\left(\mathrm{CDCl}_{3}, 300 \mathrm{MHz}\right): 2.35(\mathrm{bs}, 1 \mathrm{H}), 4.50(\mathrm{~s}, 2 \mathrm{H}), 7.29(\mathrm{~m}, 3 \mathrm{H}), 7.45(\mathrm{~m}, 2 \mathrm{H})$ \\
\hline${ }^{13} \mathrm{C} \mathrm{NMR}\left(\mathrm{CDCl}_{3}, 75 \mathrm{MHz}\right): 51.6,85.7,87.3,122.6,128.4,128.6,131.8$ \\
\hline GC-MS m/z= $132,103,77$ \\
\hline Anal. calcd for $\mathrm{C}_{9} \mathrm{H}_{8} \mathrm{NO}: \mathrm{C}, 81.79 ; \mathrm{H}, 6.10$ found $\mathrm{C}, 81.71 ; \mathrm{H}, 6.00$ \\
\hline
\end{tabular}

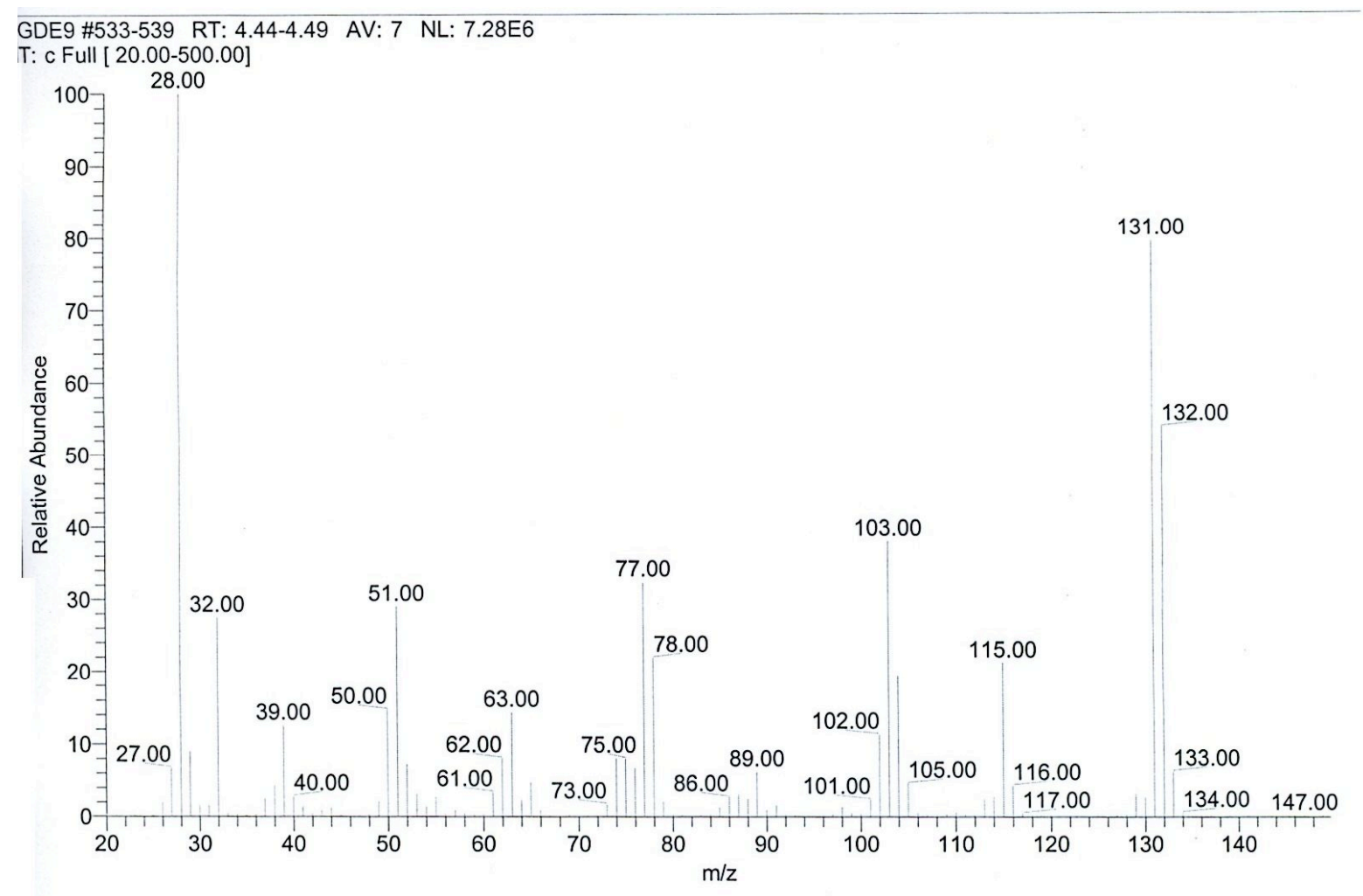




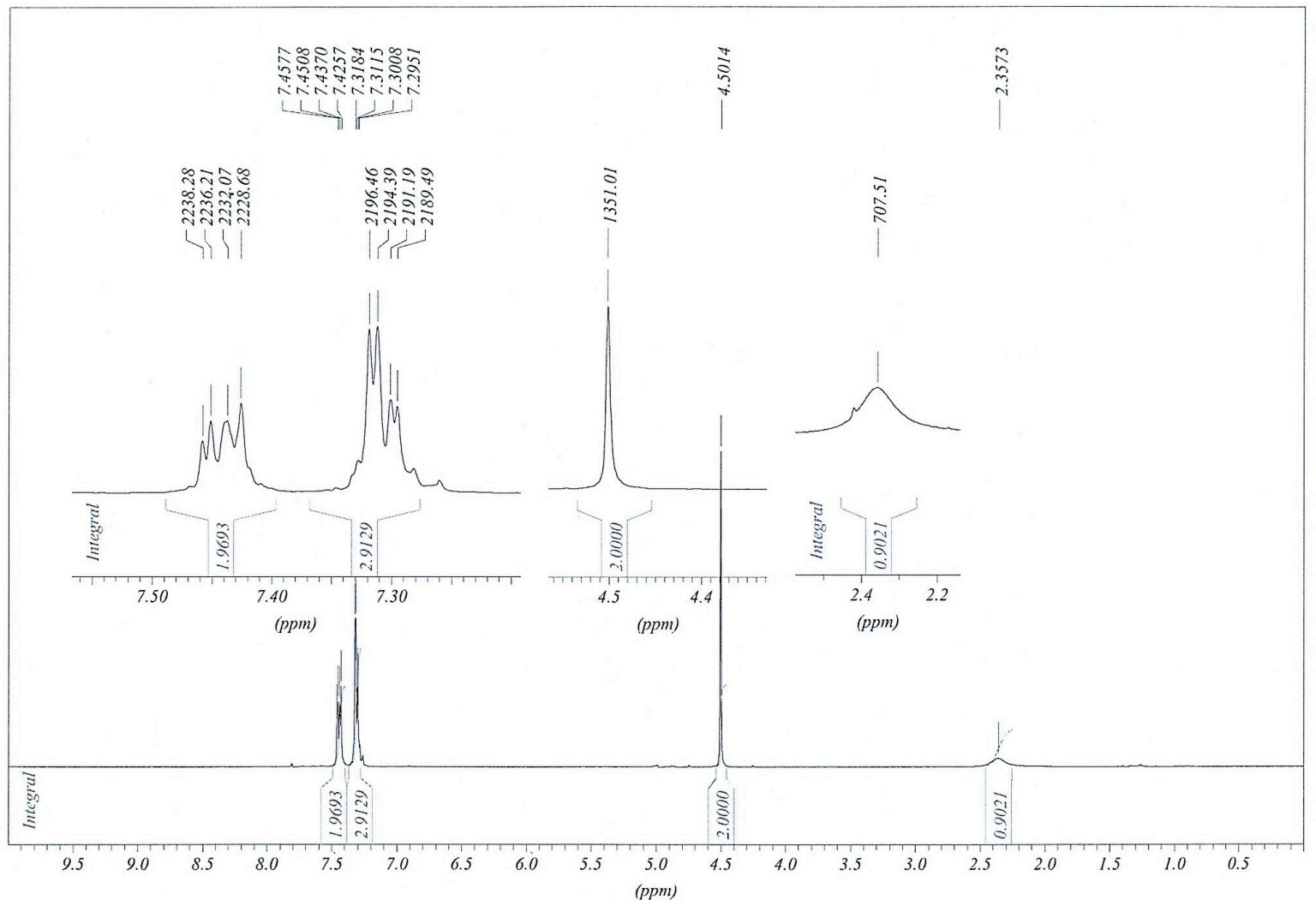

H:19-C

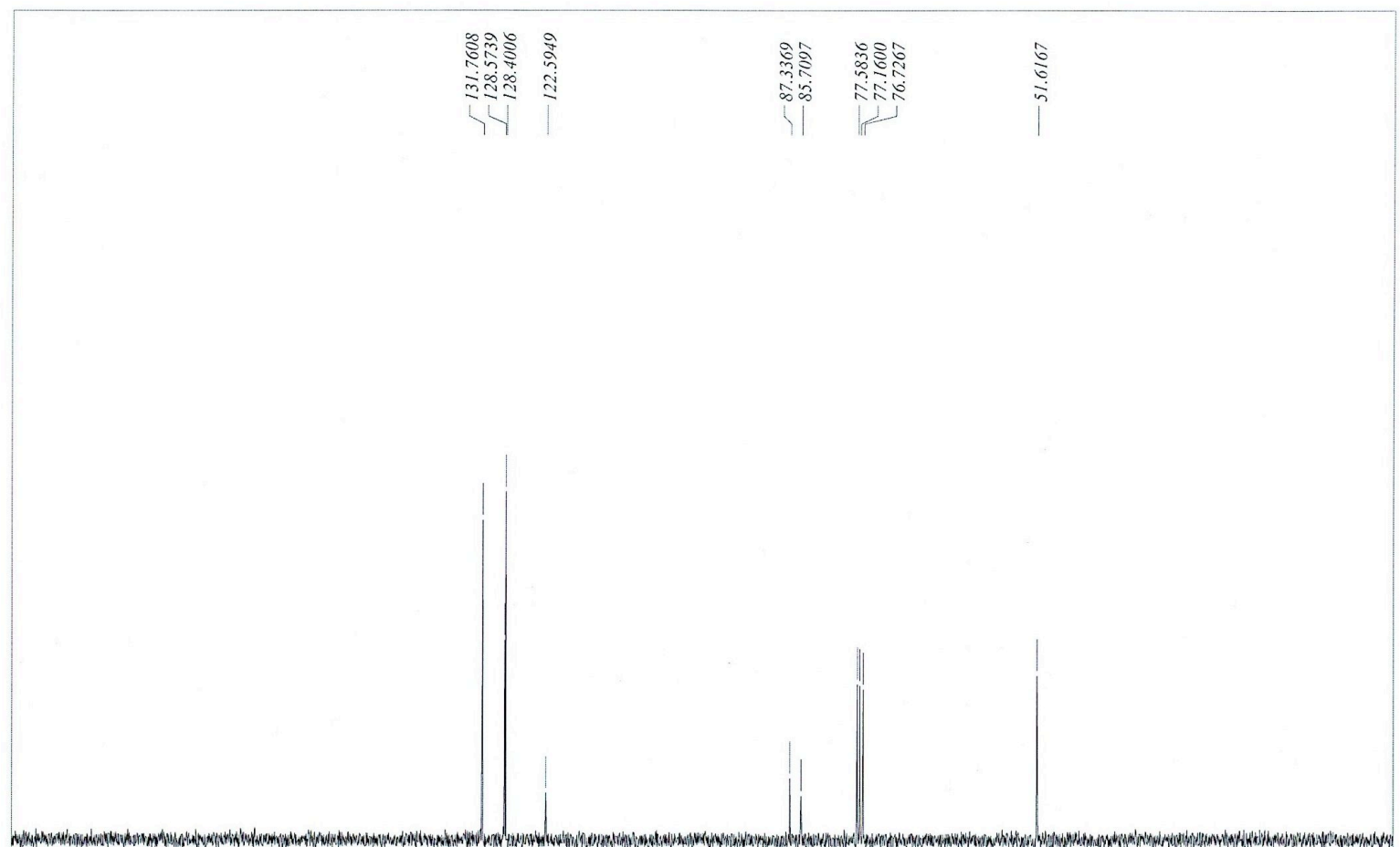

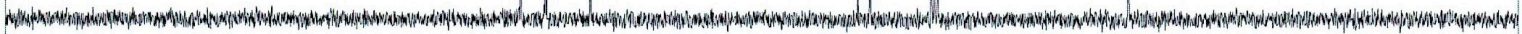

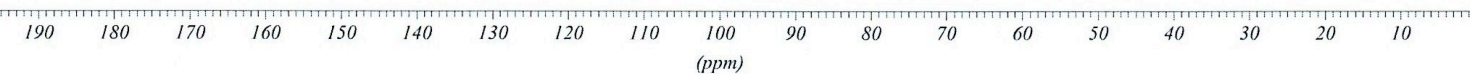


<smiles>OCC1(c2ccccc2)SCCCS1</smiles>

Mol. Formula : $\mathrm{C}_{11} \mathrm{H}_{14} \mathrm{OS}_{2}$

Mol. Weight : 226.36

Flash chromatography eluting with ethyl acetate : cyclohexane $(\mathrm{R} f=0.65)$, gave a white solid (705 mg, 93\%)

${ }^{1} \mathrm{H} \mathrm{NMR}\left(\mathrm{CDCl}_{3}, 300 \mathrm{MHz}\right): 1.95-2.14(\mathrm{~m}, 3 \mathrm{H}), 2.68$ (ddd, $\left.J=2.9 \mathrm{~Hz}, 7.4 \mathrm{~Hz}, 21.3 \mathrm{~Hz}, 2 \mathrm{H}\right)$ $2.92(\mathrm{ddd}, J=2.9 \mathrm{~Hz}, 9.0 \mathrm{~Hz}, 23.2 \mathrm{~Hz}, 2 \mathrm{H}), 4.05(\mathrm{~s}, 2 \mathrm{H}), 7.31(\mathrm{~m}, 3 \mathrm{H}), 7.85(\mathrm{~d}, J=8.3 \mathrm{~Hz}$, $2 \mathrm{H})$

${ }^{13} \mathrm{C} \mathrm{NMR}\left(\mathrm{CDCl}_{3}, 75 \mathrm{MHz}\right): 24.6,27.3,59.0,66.2,128.2,128.6,128.7,139.6$

GC-MS m/z= 226, 208, 195, 121

Anal. calcd for $\mathrm{C}_{11} \mathrm{H}_{14} \mathrm{OS}_{2}$ : C, 58.37; H, 6.23; S, 28.33 found $\mathrm{C}, 58.33 ; \mathrm{H}, 6.20 ; \mathrm{S}, 28.36$

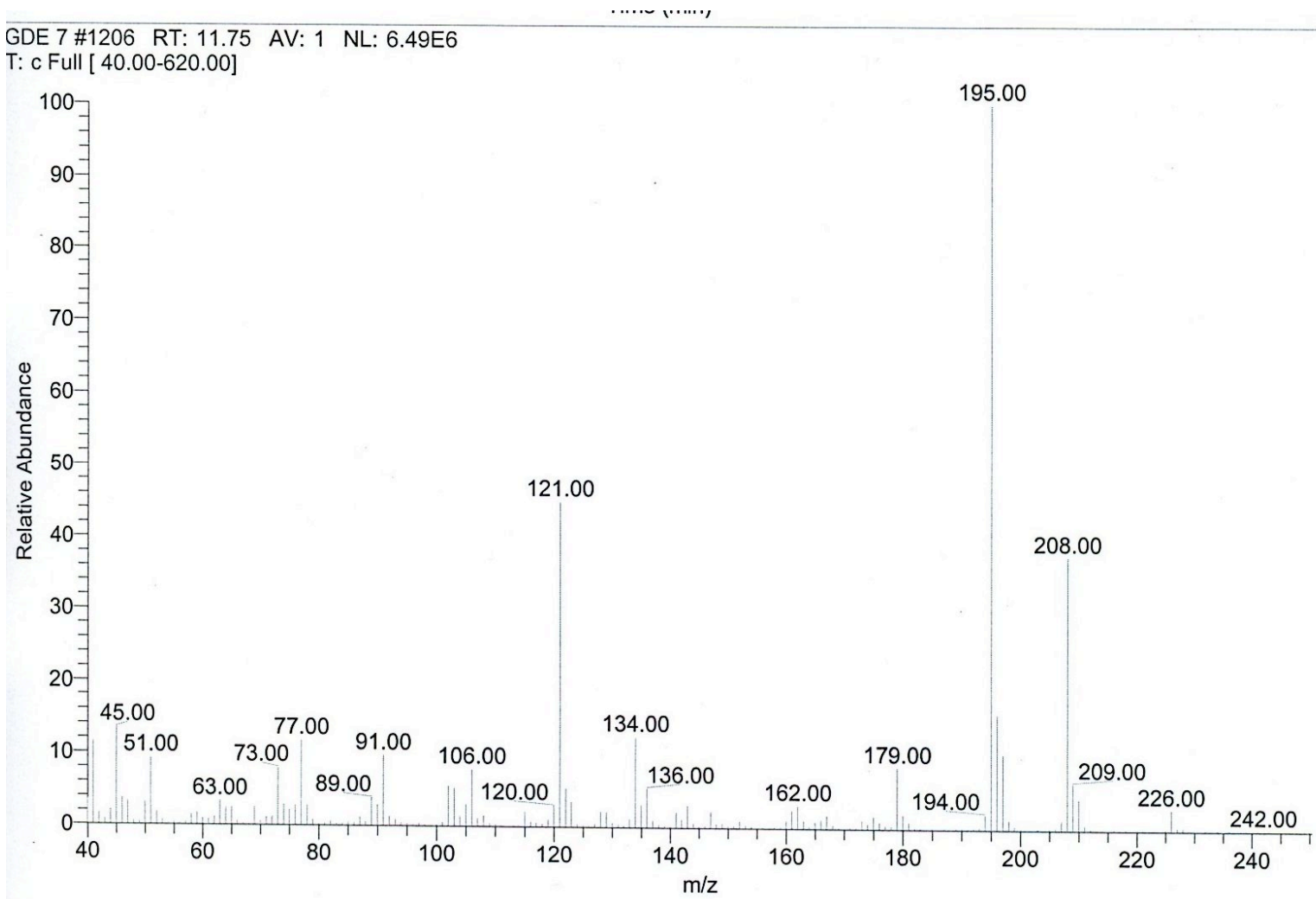




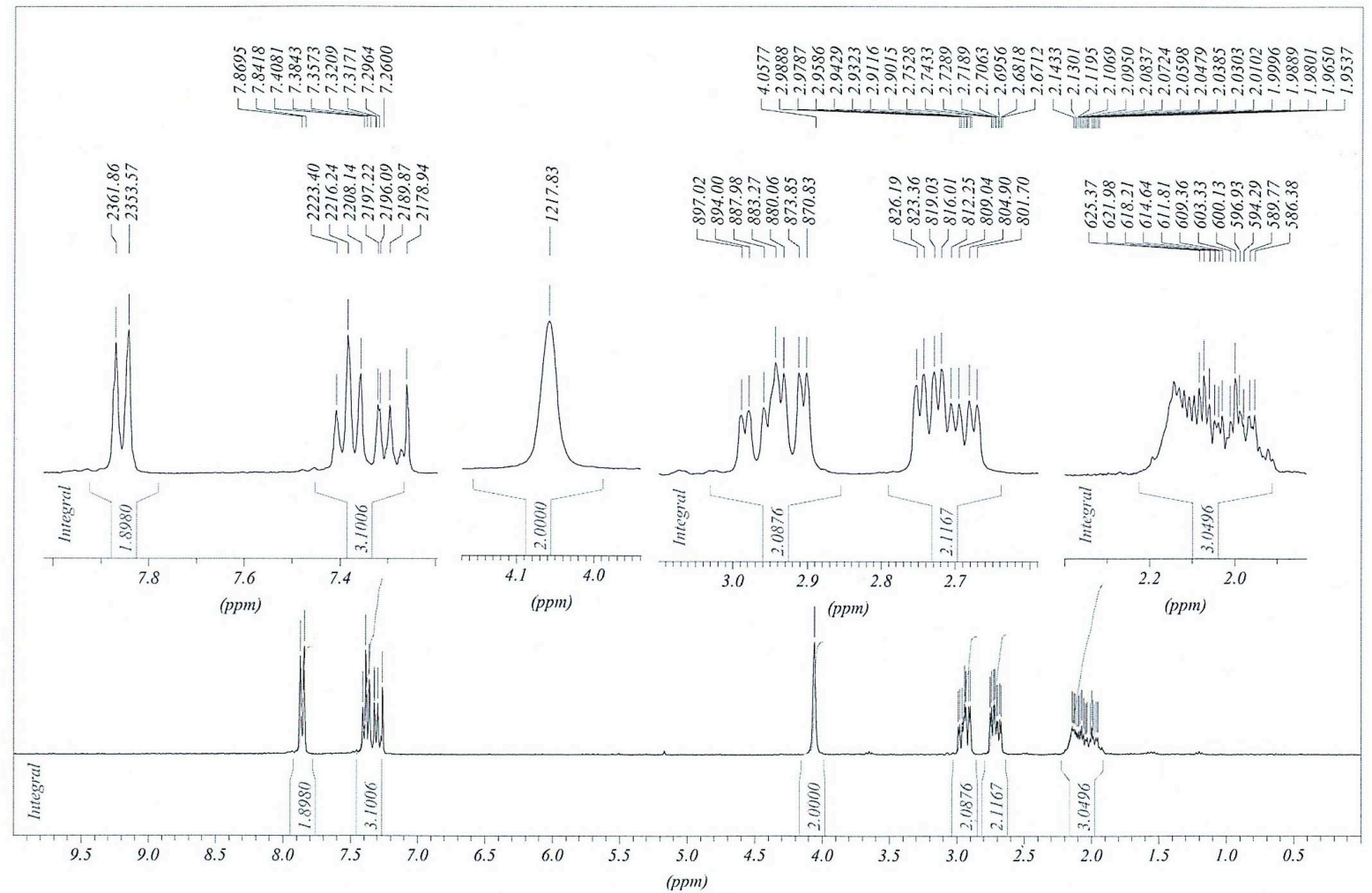

- HM7-C

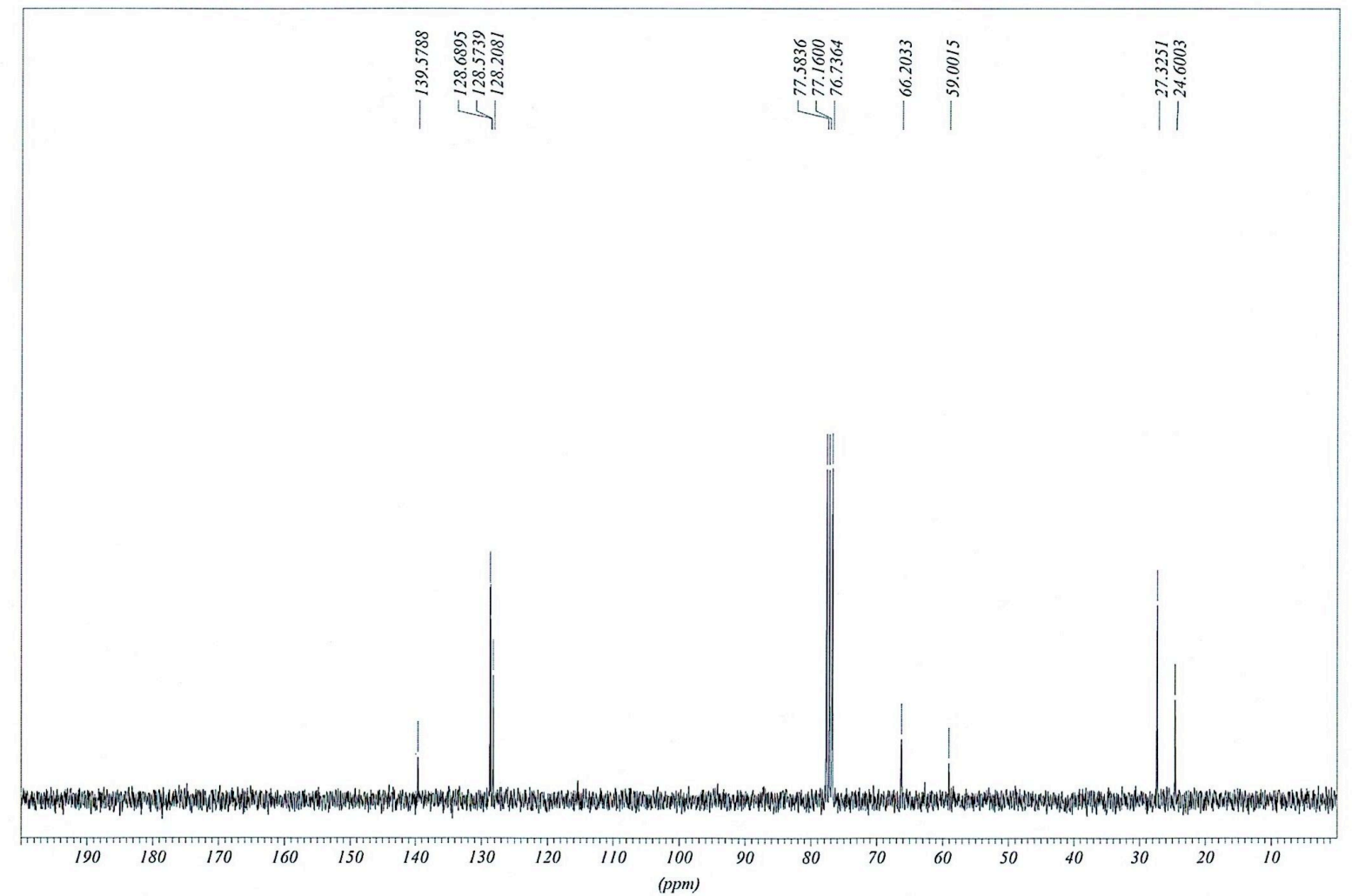




Mol. Formula : $\mathrm{C}_{9} \mathrm{H}_{9} \mathrm{NO}$
Mol. Weight : 147.17

Flash chromatography eluting with ethyl acetate : cyclohexane $1: 1(\mathrm{R} f=0.65)$ gave a colorless oil (247 mg, 50\%)

${ }^{1} \mathrm{H}$ NMR $\left(\mathrm{CDCl}_{3}, 300 \mathrm{MHz}\right): 2.53$ (bs, $\left.1 \mathrm{H}\right), 5.53$ (s, 2H), $6.46(\mathrm{~d}, J=3.2 \mathrm{~Hz}, 1 \mathrm{H}), 710(\mathrm{~m}$, 2H), $7.16(\mathrm{t}, J=8.1 \mathrm{~Hz}, 1 \mathrm{H}), 7.39(\mathrm{~d}, J=8.1 \mathrm{~Hz}, 1 \mathrm{H}), 7.57(\mathrm{~d}, J=7.7 \mathrm{~Hz}, 1 \mathrm{H})$

${ }^{13} \mathrm{C} \mathrm{NMR}\left(\mathrm{CDCl}_{3}, 75 \mathrm{MHz}\right):$ 70.0, 103.1, 109.6, 120.5, 121.3, 122.3, 127.4, 129.4, 135.7

GC-MS m/z=147, 117, 90

Anal. calcd for $\mathrm{C}_{9} \mathrm{H}_{9} \mathrm{NO}$ : C, 73.45; H, 6.16; N, 9.52 found $\mathrm{C}, 73.37 ; \mathrm{H}, 6.09 ; \mathrm{N}, 9.61$

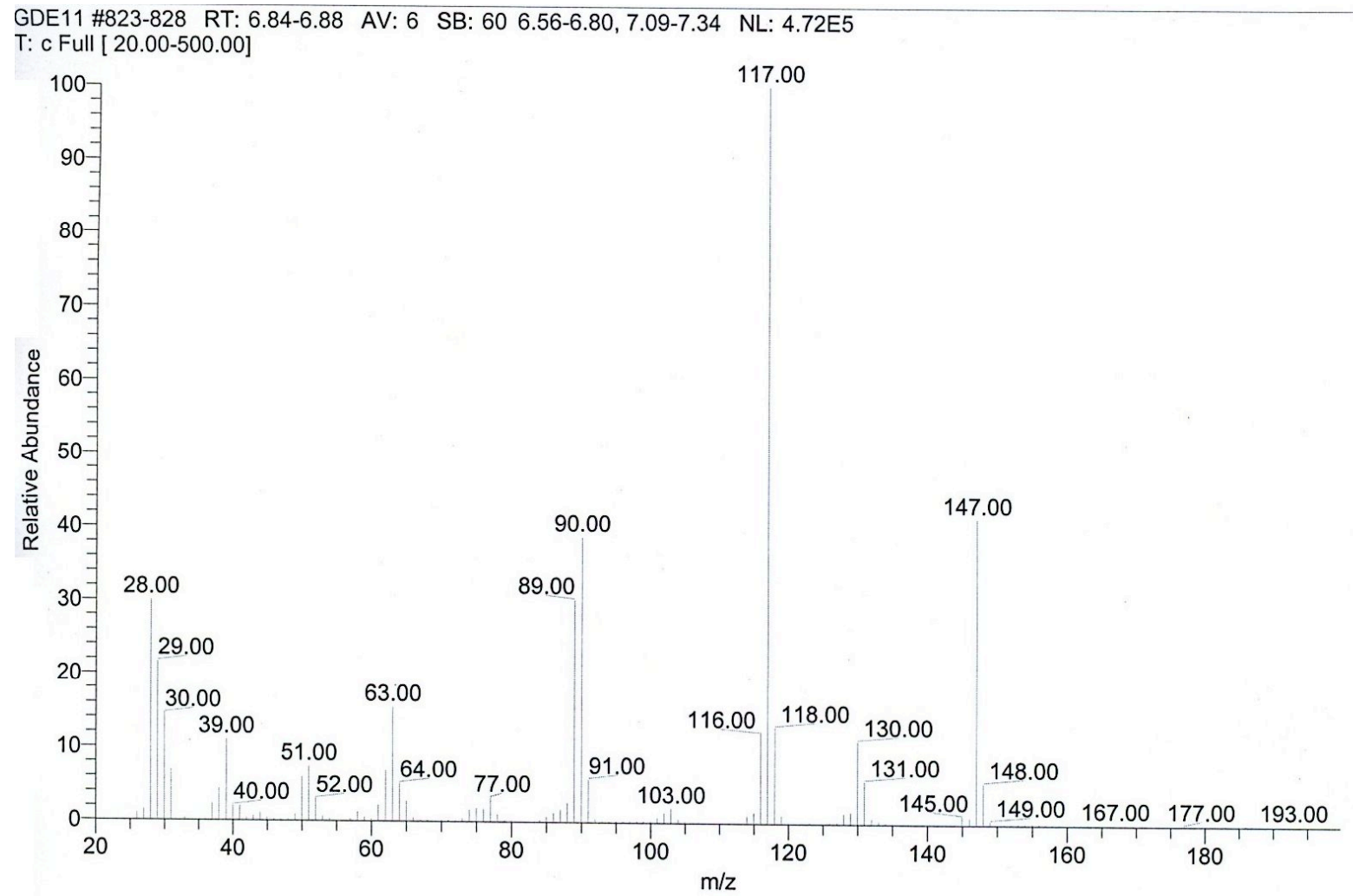




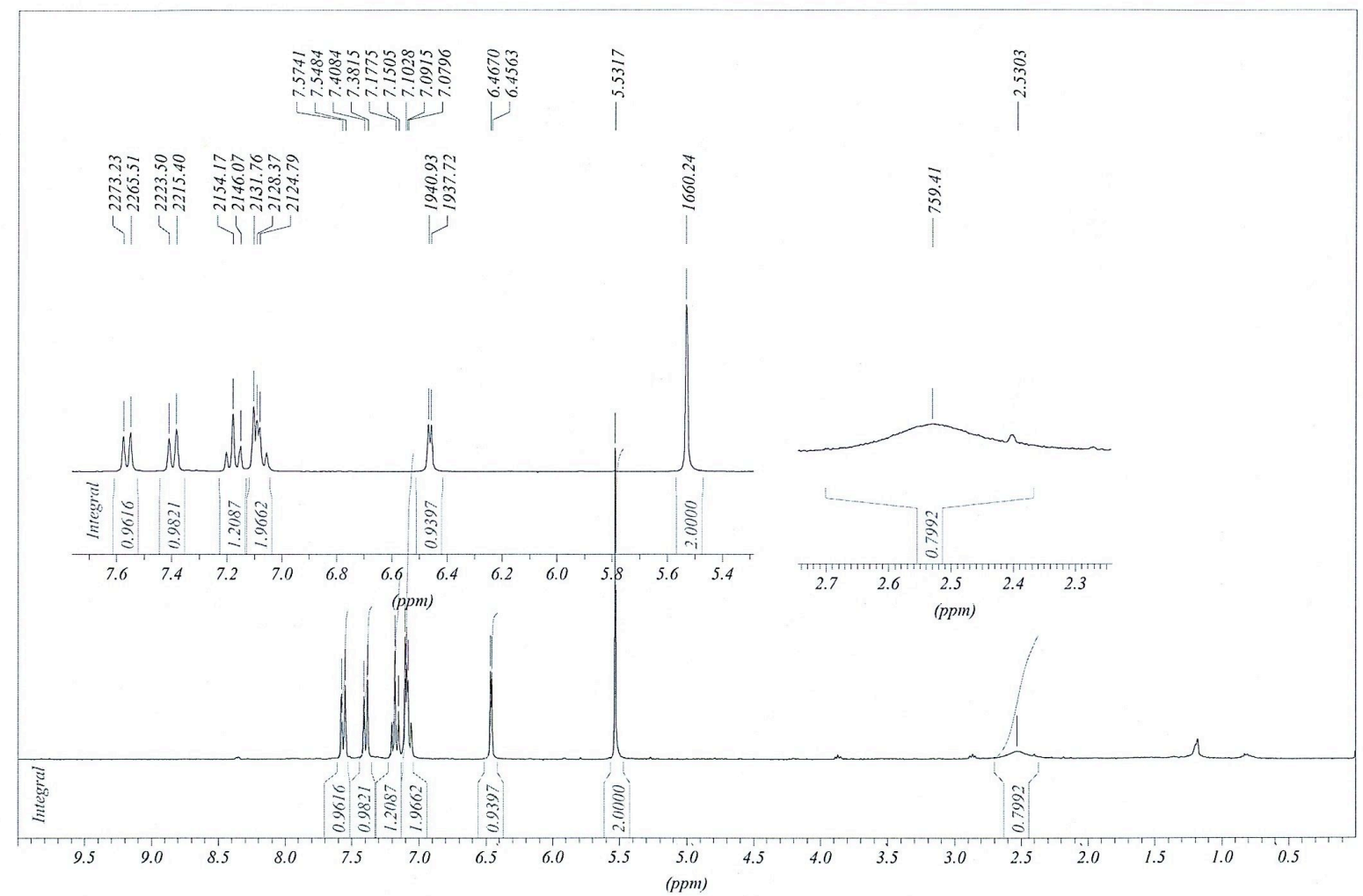

HMIII-C ?

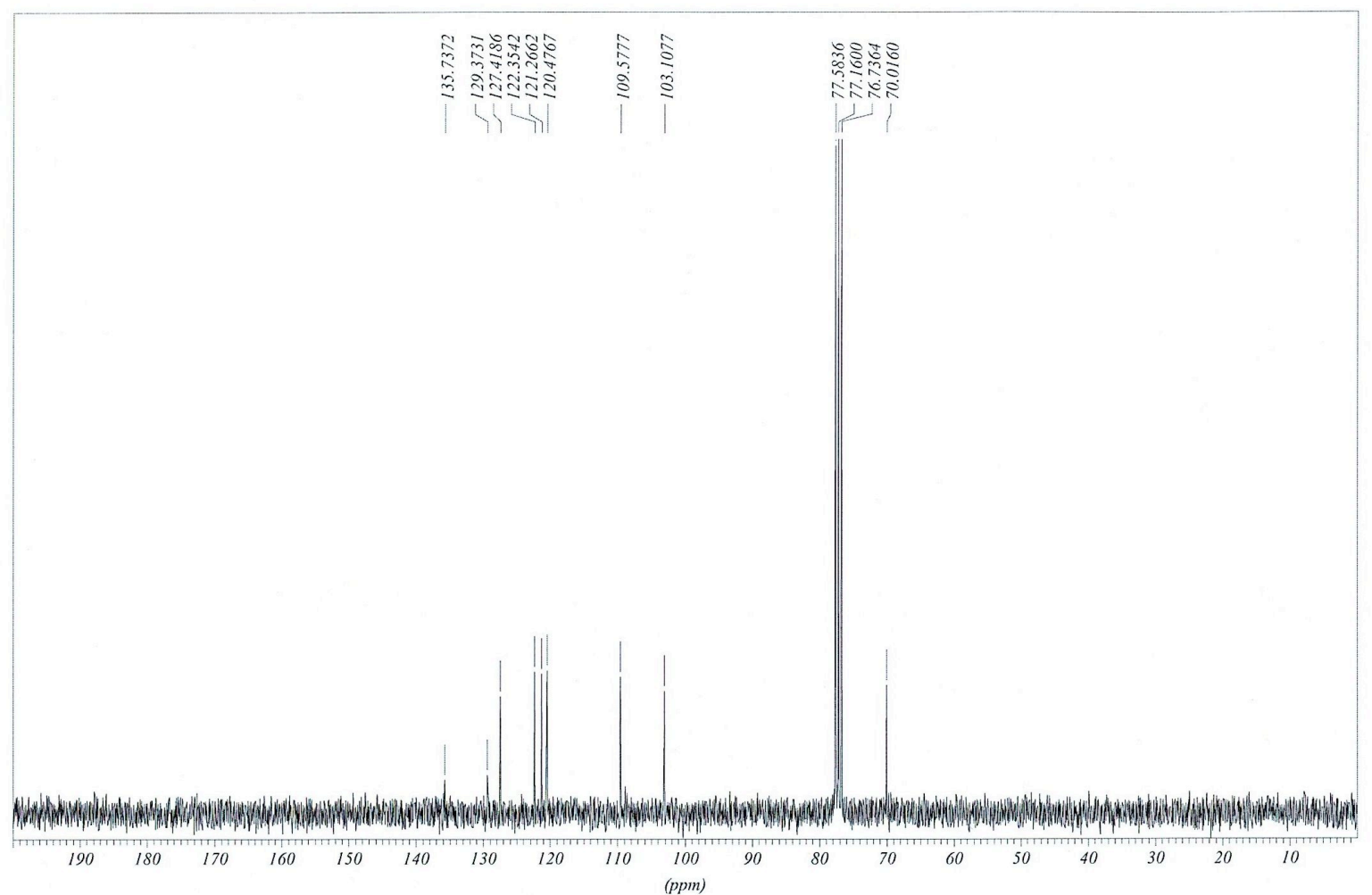




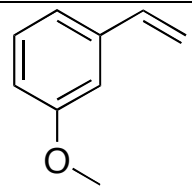

Mol. Formula : $\mathrm{C}_{9} \mathrm{H}_{10} \mathrm{O}$

Mol. Weight : 134.18

Filtration through a short plug of celite and silica gel eluting diethyl ether : cyclohexane $1: 1$ furnished 16 as a yellow oil (414 $\mathrm{mg}, 92 \%)$

${ }^{1} \mathrm{H} \mathrm{NMR}\left(\mathrm{CDCl}_{3}, 300 \mathrm{MHz}\right): 3.71(\mathrm{~s}, 3 \mathrm{H}), 5.15(\mathrm{~d}, J=10.7 \mathrm{~Hz}, 1 \mathrm{H}), 5.65(\mathrm{~d}, J=17.5 \mathrm{~Hz}$, $1 \mathrm{H}), 6.60(\mathrm{dd}, J=10.7 \mathrm{~Hz}, 17.5 \mathrm{~Hz}, 1 \mathrm{H}), 6.71(\mathrm{dd}, J=2.1 \mathrm{~Hz}, 7.9 \mathrm{~Hz}, 1 \mathrm{H}), 6.86(\mathrm{~m}, 1 \mathrm{H})$, $6.91(\mathrm{~d}, J=7.5 \mathrm{~Hz}, 1 \mathrm{H}), 7.14(\mathrm{t}, \mathrm{J}=7.9 \mathrm{~Hz}, 1 \mathrm{H})$

${ }^{13} \mathrm{C} \mathrm{NMR}\left(\mathrm{CDCl}_{3}, 75 \mathrm{MHz}\right): 54.3,110.6,112.5,113.2,117.9,128.6,135.8,138.1,158.8$

GC-MS m/z=134, 91

Anal. calcd for $\mathrm{C}_{4} \mathrm{H}_{7} \mathrm{NO}$ : C, 80.56; $\mathrm{H}, 7.51$ found $\mathrm{C}, 80.49 ; \mathrm{H}, 7.44$

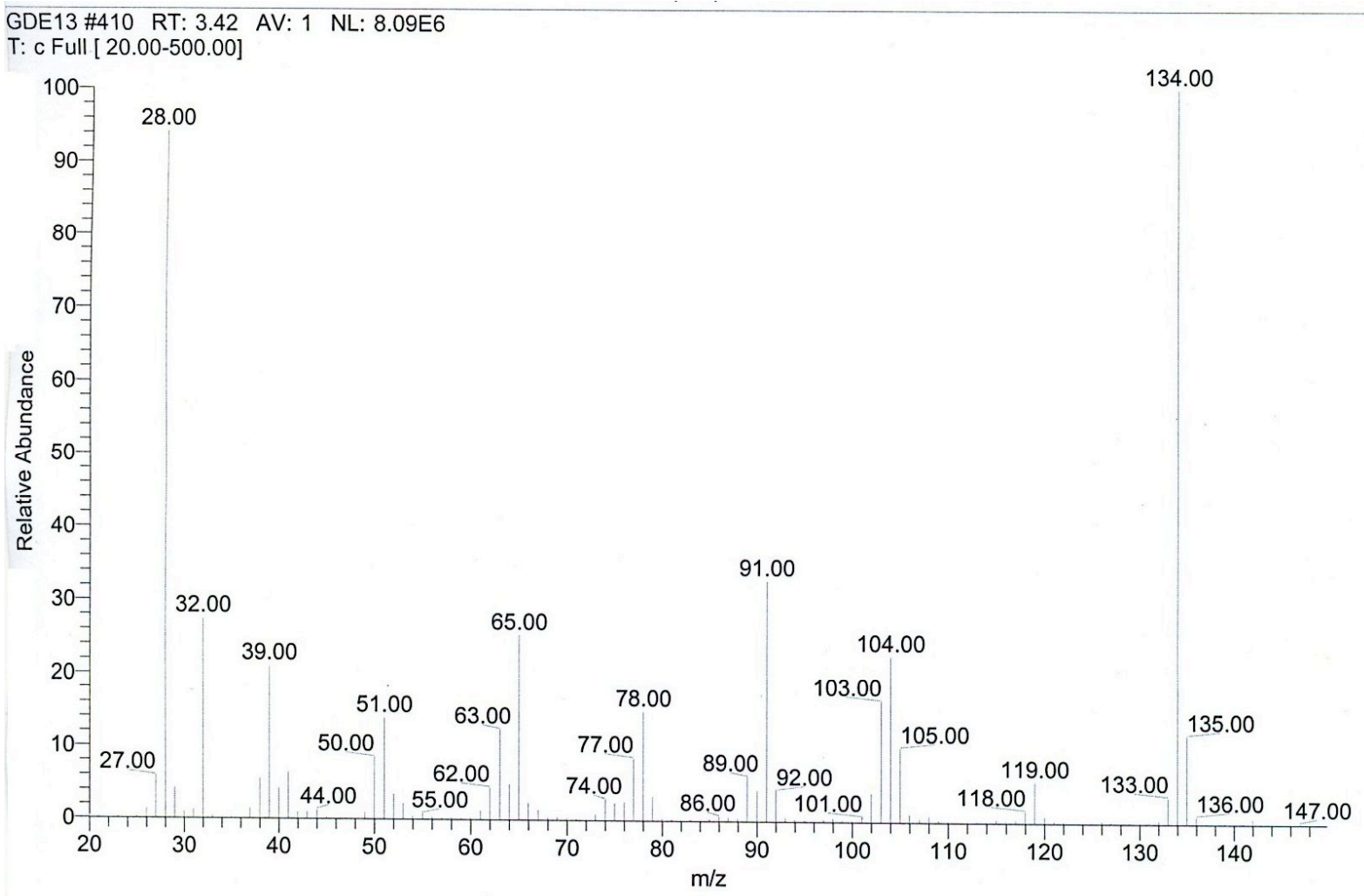




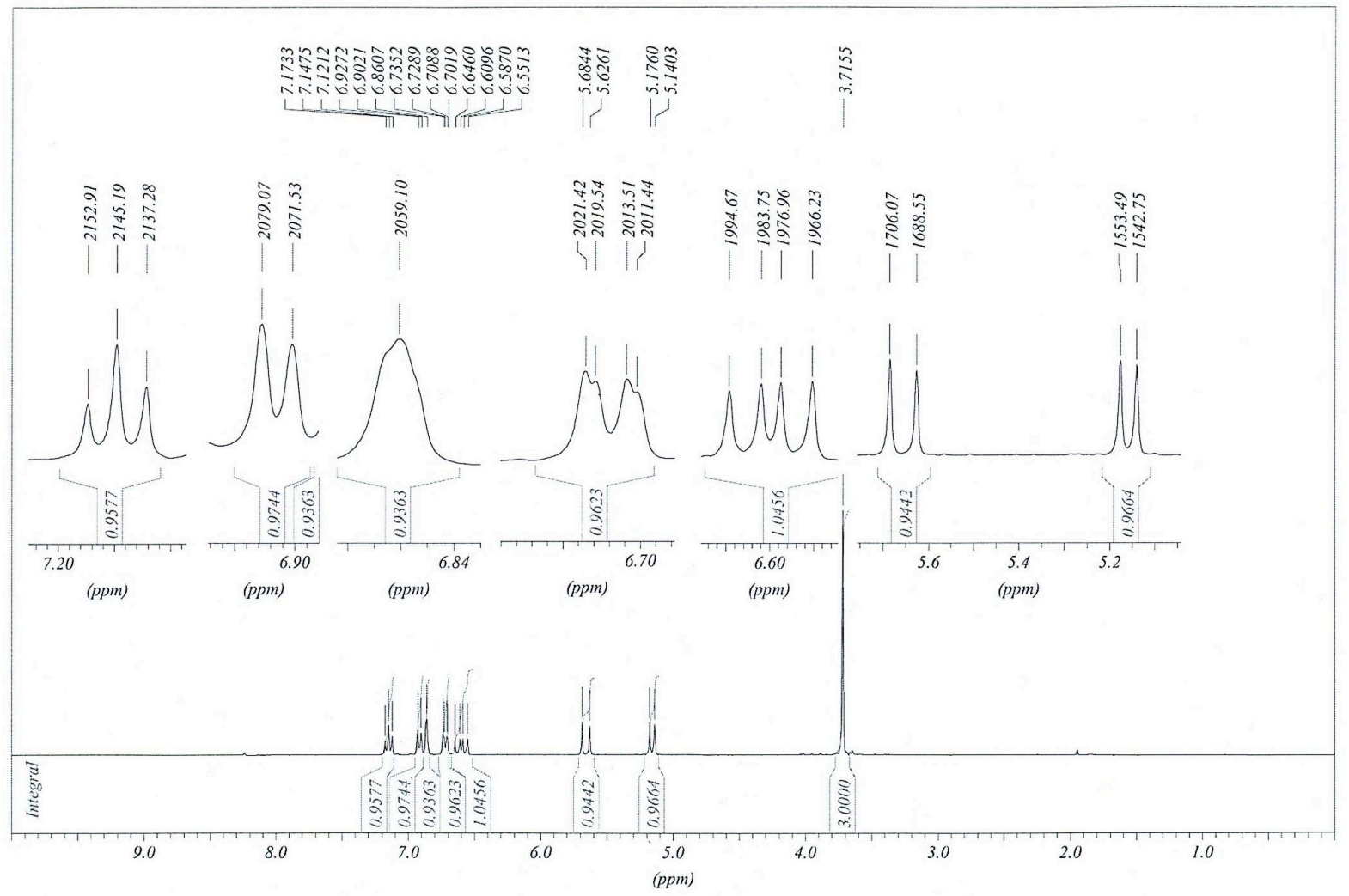

HMV $-13 \mathrm{C}$

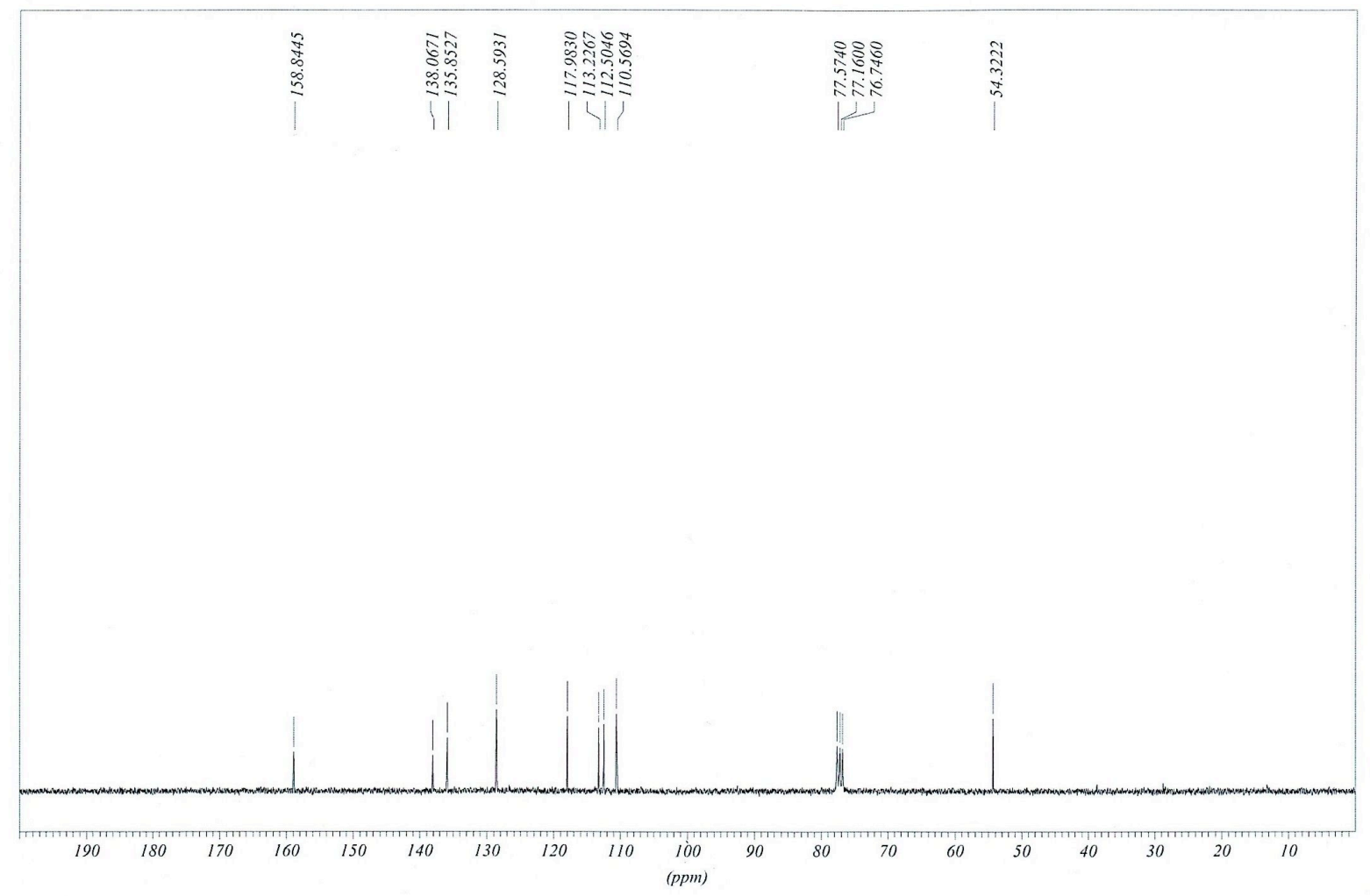

